\title{
Recent Trends in Artificial Intelligence-Assisted Coronary Atherosclerotic Plaque Characterization
}

\author{
Anjan Gudigar ${ }^{1}$, Sneha Nayak ${ }^{1}$, Jyothi Samanth ${ }^{2}$, U Raghavendra ${ }^{1, *}$, Ashwal A J ${ }^{3}$, Prabal Datta Barua ${ }^{4,5}$, \\ Md Nazmul Hasan ${ }^{6}$, Edward J. Ciaccio ${ }^{7}$, Ru-San Tan ${ }^{8,9}$ and U. Rajendra Acharya 10,11,12 (D)
}

Citation: Gudigar, A.; Nayak, S.; Samanth, J.; Raghavendra, U.; A J, A.; Barua, P.D.; Hasan, M.N.; Ciaccio, E.J.; Tan, R.-S.; Rajendra Acharya, U. Recent Trends in Artificial Intelligence-Assisted Coronary Atherosclerotic Plaque Characterization. Int. J. Environ. Res. Public Health 2021, 18, 10003. https://doi.org/10.3390/ ijerph181910003

Academic Editor: Paul B. Tchounwou

Received: 9 August 2021

Accepted: 17 September 2021

Published: 23 September 2021

Publisher's Note: MDPI stays neutral with regard to jurisdictional claims in published maps and institutional affiliations.

Copyright: (c) 2021 by the authors. Licensee MDPI, Basel, Switzerland. This article is an open access article distributed under the terms and conditions of the Creative Commons Attribution (CC BY) license (https:/ / creativecommons.org/licenses/by/ $4.0 /)$.
1 Department of Instrumentation and Control Engineering, Manipal Institute of Technology, Manipal Academy of Higher Education, Manipal 576104, India; anjan.gudigar@manipal.edu (A.G.); sneha.nayak@manipal.edu (S.N.)

2 Department of Cardiovascular Technology, Manipal College of Health Professions, Manipal Academy of Higher Education, Manipal 576104, India; samanth.jyothi@manipal.edu

3 Department of Cardiology, Kasturba Medical College, Manipal Academy of Higher Education, Manipal 576104, India; ashwal.aj@manipal.edu

4 School of Management \& Enterprise, University of Southern Queensland, Toowoomba, QLD 4350, Australia; Prabal.Barua@usq.edu.au

5 Faculty of Engineering and Information Technology, University of Technology Sydney, Sydney, NSW 2007, Australia

6 Department of Cardiology, Ad-Din Medical College Hospital, Dhaka 1217, Bangladesh; nazmulhasan45cmc@gmail.com

7 Department of Medicine, Division of Cardiology, Columbia University Medical Center, New York, NY 10032, USA; edwardciaccio@gmail.com

8 Department of Cardiology, National Heart Centre Singapore, Singapore 169609, Singapore; tan.ru.san@singhealth.com.sg

9 Duke-NUS Medical School, Singapore 169857, Singapore

10 School of Engineering, Ngee Ann Polytechnic, Clementi 599489, Singapore; Rajendra_Udyavara_ACHARYA@np.edu.sg

11 Department of Biomedical Informatics and Medical Engineering, Asia University, Taichung 41354, Taiwan

12 Department of Biomedical Engineering, School of Science and Technology, Singapore University of Social Sciences, Singapore S599494, Singapore

* Correspondence: raghavendra.u@manipal.edu

Abstract: Coronary artery disease is a major cause of morbidity and mortality worldwide. Its underlying histopathology is the atherosclerotic plaque, which comprises lipid, fibrous and-when chronic - calcium components. Intravascular ultrasound (IVUS) and intravascular optical coherence tomography (IVOCT) performed during invasive coronary angiography are reference standards for characterizing the atherosclerotic plaque. Fine image spatial resolution attainable with contemporary coronary computed tomographic angiography (CCTA) has enabled noninvasive plaque assessment, including identifying features associated with vulnerable plaques known to presage acute coronary events. Manual interpretation of IVUS, IVOCT and CCTA images demands scarce physician expertise and high time cost. This has motivated recent research into and development of artificial intelligence (AI)-assisted methods for image processing, feature extraction, plaque identification and characterization. We performed parallel searches of the medical and technical literature from 1995 to 2021 focusing respectively on human plaque characterization using various imaging modalities and the use of AI-assisted computer aided diagnosis (CAD) to detect and classify atherosclerotic plaques, including their composition and the presence of high-risk features denoting vulnerable plaques. A total of 122 publications were selected for evaluation and the analysis was summarized in terms of data sources, methods-machine versus deep learning-and performance metrics. Trends in AI-assisted plaque characterization are detailed and prospective research challenges discussed. Future directions for the development of accurate and efficient CAD systems to characterize plaque noninvasively using CCTA are proposed. 
Keywords: artificial intelligence; computer aided diagnosis; coronary angiography; coronary artery disease; coronary computed tomographic angiography; intravascular optical coherence tomography; intravascular ultrasound

\section{Introduction}

Coronary artery disease is a major cause of morbidity and mortality worldwide. Its underlying histopathology is the formation of atherosclerotic plaques within the wall lining of the coronary artery tree [1]. Chronic morphological adaptation of the plaque is characterized by progressive necrotic changes and calcification [2]. Initially, the plaque is nonobstructive and the patient is asymptomatic. Accumulation of plaque components increases the plaque atheroma volume, which encroaches on the coronary lumen $[3,4]$. As the coronary luminal area becomes reduced, blood flow delivery to heart muscles becomes compromised at high-demand stress states, i.e., myocardial ischemia, which manifests as angina [5]. Progressive arterial wall remodeling alters plaque composition and surface, rendering it vulnerable to erosion and even rupture [6]. This incites chain chemical reactions that precipitate acute thrombosis, which occludes the coronary lumen causing myocardial infarct [7].

Atherosclerotic plaques comprise heterogeneous components such as extracellular lipids, collagen fibers (fibrous tissue), loose collagen fibers with lipid accumulation (mixed tissue) and-when chronic — compact calcium crystal deposits (calcified tissue) [8]. The American Heart Association grades the atherosclerotic plaque into classes with incremental histo-morphological complexity [9]: Type I, normal wall thickness or minimal intimal thickening, some macrophages with little lipid deposits or foam cells; Type II, additional smooth muscle cells with little lipid deposits, T-lymphocytes and rare mast cells; Type III (pre-atheroma), increased extracellular lipid; Type IV, (atheroma), confluent lipid deposits (lipid core) covered mainly by intima; Type $\mathrm{V}$, increasing lipid deposits with well-defined fibrous (collagen) cap (fibroatheroma, type Va) or predominant calcifications (calcific, types $\mathrm{V} \mathrm{b}$ and $\mathrm{c}$ ); Type VI, complicated lesion with disruption of lesion surface, hematoma or hemorrhage, and thrombotic deposits have developed [10].

Intravascular ultrasound (IVUS) and intravascular optical coherence tomography (IVOCT) performed via catheter during invasive coronary angiography (CAG) possess high spatial resolution and signal contrast, and are the reference standards for in vivo near-field characterization of individual atherosclerotic plaques. Based on the constituents, atheromatous plaques can be stratified as stable or vulnerable. The former is characterized by heavy calcification, fibrotic tissue, and small lipid pools. In contrast, the latter contains a large lipid pool (necrotic core) with or without spotty calcification, and is covered by a thin fibrous cap that is soft in nature and prone to rupture-the thin-cap fibroatheroma (TCFA). Post-processing techniques such as virtual histology intravascular ultrasound (VH-IVUS) [11] can enhance visualization of early pathology, e.g., intimal thickening (PIT), as well as high-risk lesions such as TCFA and calcified TCFA. Similarly, IVOCT can characterize human atherosclerotic plaques into fibrous, fibrocalcific and lipid rich types with histology-like accuracy [12]. Using IVOCT, TCFA was more frequently seen in patients presenting with acute coronary syndrome than those with stable angina [13].

Noninvasive coronary computed tomographic angiography (CCTA) can also characterize atherosclerotic plaques albeit with less fine spatial resolution than invasive IVUS and IVOCT. Of advantage, CCTA can survey the entire coronary tree within a single three-dimensional image dataset and possesses high sensitivity for calcium deposition. Coronary plaques can thus be categorized as noncalcified, partially calcified and calcified, and the severity of calcification quantitated. Using advanced analysis, lipid and fibrotic components in noncalcified plaques can also be identified [14].

Manual identification and characterization of coronary plaques demands clinical expertise and high time costs. It is also dependent on image quality, which is easily affected 
by speckle noise. These reasons motivate the development of computer aided diagnosis (CAD) systems for automated image processing as well as coronary plaque identification and characterization on both invasive [15] and noninvasive image readouts [16]. With the help of $\mathrm{CAD}$, image quality can be improved, which helps in the accurate interpretation of results. Use of CAD by physicians can be considered as a countercheck to reduce diagnostic error and physician workload. Algorithms can enhance physician performance by increasing the ease and efficiency of decision-making. Given the importance of early diagnosis and risk stratification, accurate, and efficient automated diagnostic tools for coronary plaque characterization based on non-invasive imaging modalities like CCTA are desirable. In this review, our aims were as follows:

- To compare manual grading systems for plaque characterization with various imaging modalities;

- To analyze state-of-the-art artificial intelligence (AI) techniques to characterize plaque;

- To discuss the results and roles of different techniques for plaque characterization; and

- To highlight potential research gaps and future research directions related to plaque characterization using CAD.

The remainder of the paper is structured as follows: description of article selection process is in Section 2; review of commonly used imaging modalities for detecting and grading coronary plaques appears in Section 3; overview of different machine learning (ML) and deep learning (DL) techniques for coronary plaque detection and classification are in Section 4; comparison of the performance of various CAD techniques when applied to different imaging modalities as well as discussion of current challenges, potential research gaps, and future directions are found in Section 5; and study conclusions are in Section 6.

\section{Review Process}

The review process was carried out based on the procedures and guidelines presented in PRISMA [17]. We performed a search of the following databases, IEEEXplore, PubMed, Springer, Scopus, and Google Scholar, for articles published between 1 January 1995 and 25 June 2021 using the keywords 'deep learning AND plaque detection', 'machine learning AND plaque detection', 'computer aided diagnostic tools', 'coronary plaque segmentation', 'computed tomography angiography', 'coronary plaque detection', 'intravascular ultrasound', 'intravascular optical coherence tomography', 'atherosclerotic plaque', 'plaque morphology', 'support vector machine AND plaque classification' and 'convolutional neural networks AND plaque detection'.

To identify the most suitable and eligible articles: These articles were further filtered using inclusion/exclusion criteria. We included prospective studies that compared various imaging modalities such as CCTA, IVOCT and IVUS in the diagnosis and characterization of coronary atherosclerotic plaques. In addition, studies that employed various ML and DL to segment plaque regions from the image dataset, as well as to detect and classify coronary plaque type, were considered. We also considered clinical studies that focused on coronary plaque detection, grading, and characterization including coronary calcification. Studies related to image acquisition technique, non-coronary vascular assessment, coronary anomalies, coronary artery stenosis severity, and only animals were excluded. The selected articles comprised those with medical and technical contents (see Figure 1). The final selection of 61 medical and 61 technical publications from 154 and 116 screened, respectively, were all published in the last fifteen years. 


\section{Manuscript analysis}

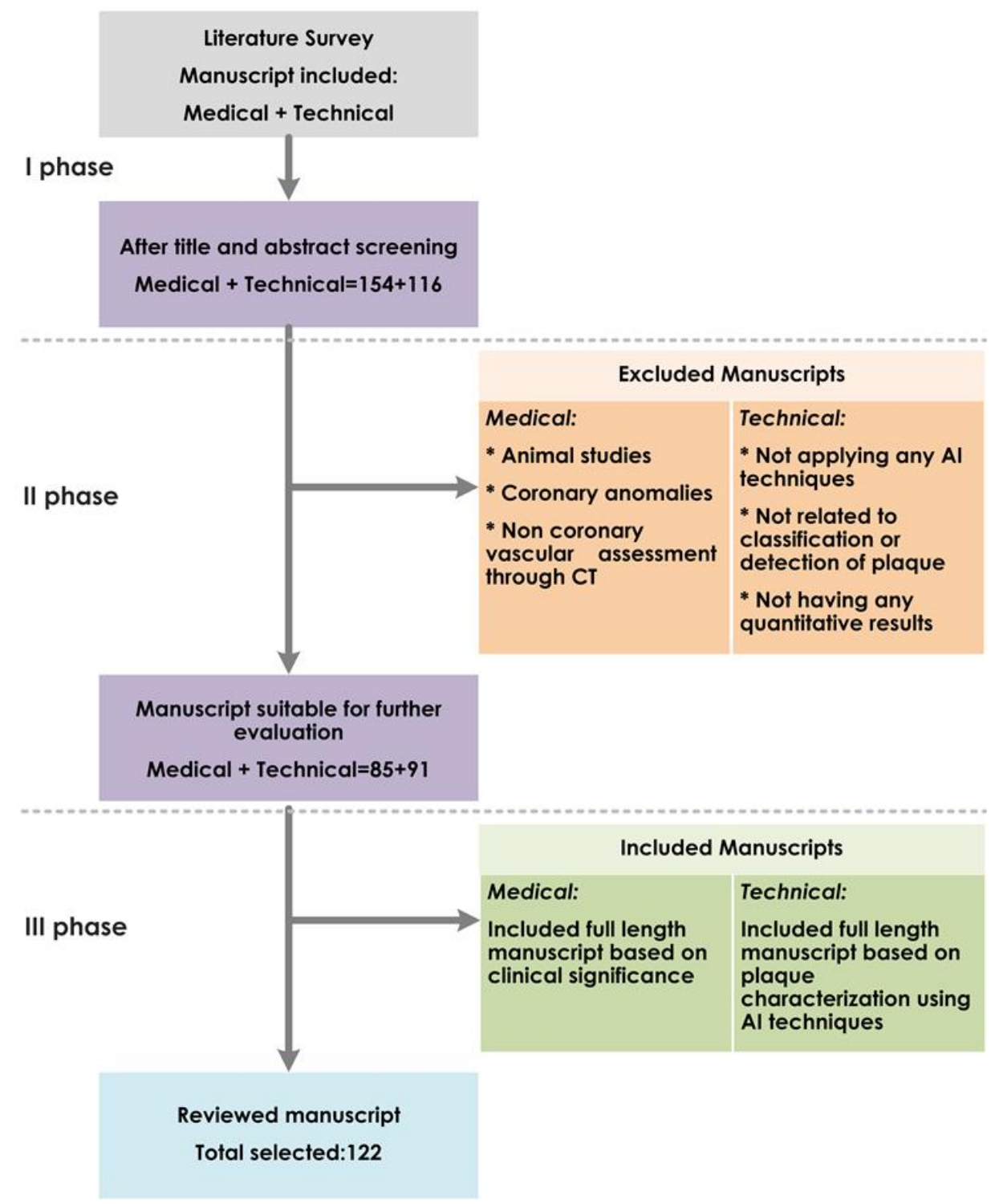

Figure 1. Workflow of study strategy and manuscript selection process.

\section{Current Coronary Artery Disease Detection Modalities and Grading System}

Several invasive and non-invasive modalities are employed in clinical practice for the identification and assessment of coronary plaques [18], e.g., IVUS (including VH-IVUS), IVOCT, CCTA (see Figure 2), positron emission tomography, magnetic resonance imaging, and near-infrared spectroscopy.

Invasive CAG is the gold standard for coronary artery disease diagnosis based on detection of coronary lumen stenosis but is of limited utility for plaque characterization beyond outlining intracoronary thrombus associated with ruptured atherosclerotic plaque. Nevertheless, invasive CAG is a prerequisite for plaque characterization with IVUS and IVOCT. IVUS is the gold standard for assessment of the atherosclerotic coronary plaque $[19,20]$. A miniaturized ultrasound probe is introduced intra-luminally via an intravascular catheter to the coronary lesion to obtain near-field two-dimensional crosssectional high-resolution real-time images of the coronary lumen and atherosclerotic plaque with high echogenic grey-scale signal contrast [21,22]. IVUS is able to distinguish the intimal, medial, and adventitial layers of the arterial wall as well as various components of the atherosclerotic plaque, including the presence of dense calcium (DC), necrotic core (NC), 
fibrotic tissue (FT), and fibrofatty tissue (FFT) [23,24]. It yields comprehensive quantitative information on lumen size, plaque components and presence of vulnerable plaque features or complications, e.g., TCFA or plaque surface rupture, which herald an imminent acute coronary event [25]. VH-IVUS uses spectral analysis of the backscattered grey-scale IVUS radiofrequency signals to augment tissue contrast among the various plaque components [26]. The results are displayed in color codes, which enhances visualization of pathology. Instead of sound waves, IVOCT exploits light waves to produce near-field plaque images with superior spatial resolution (approximately $15 \mu \mathrm{m}$ versus $150 \mu \mathrm{m}$ for IVUS) and tissue discrimination but less imaging depth (few millimeters versus five to ten millimeters for IVUS), which may unveil surface details not appreciated on IVUS [27]. Calcified plaque identification as well as discrimination between fibrous and lipid tissues are better with IVOCT than with IVUS.

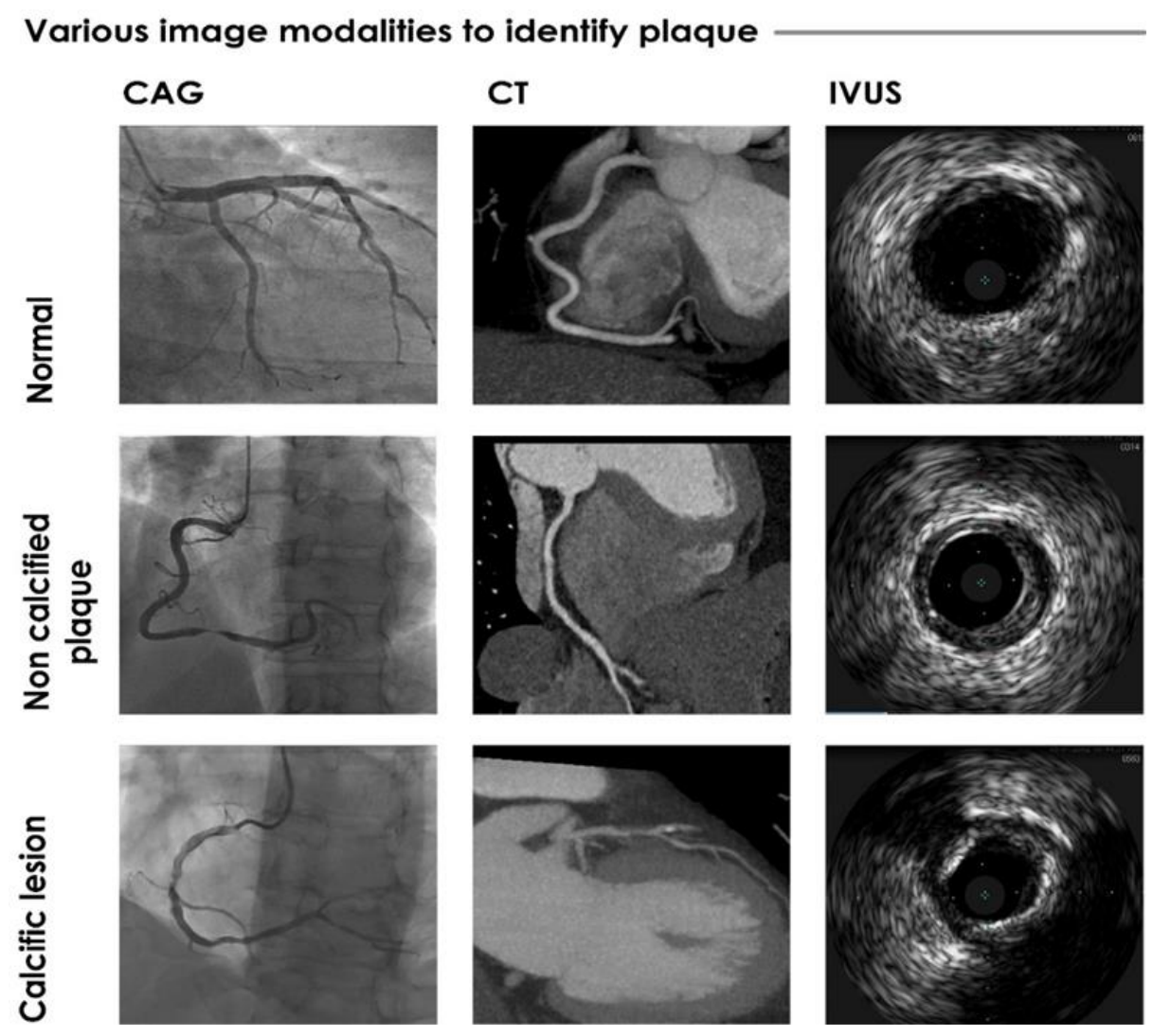

Figure 2. Sample images from invasive CAG, CT, and IVUS imaging techniques.

Multi-slice CCTA has evolved in the past two decades to become the dominant noninvasive imaging technique for coronary artery disease diagnosis [28,29], which provides information on coronary anatomy, calcification, location of stenosis and occlusion, as well as plaque morphology [8-10,30,31]. While CCTA image acquisition relies on ionizing radiation, the dose requirement has gradually been reduced by $30-50 \%$ through use of prospective electrocardiography-gated dose-dependent acquisition protocols on contemporary scanners [32]. While CCTA tends to overestimate coronary lesion severity compared with invasive CAG and IVUS [33], major trials that compared CCTA against quantitative coronary angiography with invasive CAG showed high sensitivity of $85-99 \%$ and fair specificity of $64-90 \%$ [34-37] for prediction of coronary artery stenosis. The clinical utility is highest in low-intermediate risk populations [38] rather than high-risk groups in which CAG with the option of therapeutic intervention at the same setting confers advantage. Calcified coronary plaques are readily visualized on computed tomography (CT) and the burden of coronary calcium, i.e., coronary calcium score, is an independent predictor of long-term outcome including incident death, myocardial infarction, and need for revascu- 
larization. However, the absence of coronary calcification does not imply the absence of coronary artery disease. Non-calcified plaques, significant coronary stenoses $(50 \%)$, and $>70 \%$ stenoses can be present in $11-13 \%, 0.9-3.7 \%$, and $0.4-1.5 \%$ of patients with a coronary calcium score of zero [39-41].

Using early four-detector CT scanners, Becker et al. [42] showed that CCTA could effectively distinguish between histologically validated lipid-rich and fibrous-rich noncalcified plaques, with significant CT attenuation differences between the two. Technological advances in CT hardware (e.g., 256 or more multi-slice scanners), acquisition and post-processing have improved image resolution and the ability to detect plaques as well as characterize their composition and features, including high-risk non-calcified plaques [43-49]. CCTA has high positive predictive value (PPV) for identifying vulnerable plaques that are at risk for rupture [34,50]. While IVUS offers superior resolution compared with CCTA for quantitative plaque characterization [51-53], CCTA offers expedient comprehensive assessment of the entire coronary artery tree in a single acquisition, and is non-invasive. In a meta-analysis of 33 studies [54], CCTA demonstrated an excellent 90\% sensitivity and $92 \%$ specificity prediction rate for coronary artery disease when referenced against IVUS.

\section{Artificial Intelligence (AI): Characterization of Plaque}

$\mathrm{AI}$ enables extraction and processing of quantitative information using human-like intelligence systems [55-59]. In recent years, the increase in generation and availability of medical imaging data has spawned research into clinical applications of AI [60-65]. Early detection of coronary plaque accumulation is important to avert complications, and many ML and DL CAD algorithms have been proposed for the automatic detection and classification of coronary plaques. CAD tools can improve clinical workflow efficiency by increasing the accuracy and timeliness of image interpretation. Figure 3 shows typical processes and techniques involved in ML and DL models for detection and classification of coronary plaques, as well as the standard performance metrics.

\subsection{ML and DL Techniques in CAD}

As described above, IVUS, IVOCT, and CCTA are the imaging modalities that can be used to characterize coronary atherosclerotic plaques. Research and development of CAD tools for plaque characterization have relied on image datasets from private [66-68] or public sources [69-73]. Some of the latter are available only upon request [25,72].

Machine Learning Techniques

ML uses various algorithms for the following key steps: preprocessing and segmentation; feature extraction; dimensionality reduction or feature ranking; and classification. Image preprocessing is a crucial preliminary step as it enhances the image quality or resolution using appropriate filters [74-76]. Extraction of the correct region of interest (ROI) is the necessary initial step for coronary plaque detection before further characterization. In order to detect or characterize the plaque, suitable features are generated using feature extraction algorithms and subsequently processed for dimensionality reduction. The extracted features may be ranked using feature ranking measures, which are then sent to a classifier to categorize the plaque type. 


\section{Al Models to characterize plaque}

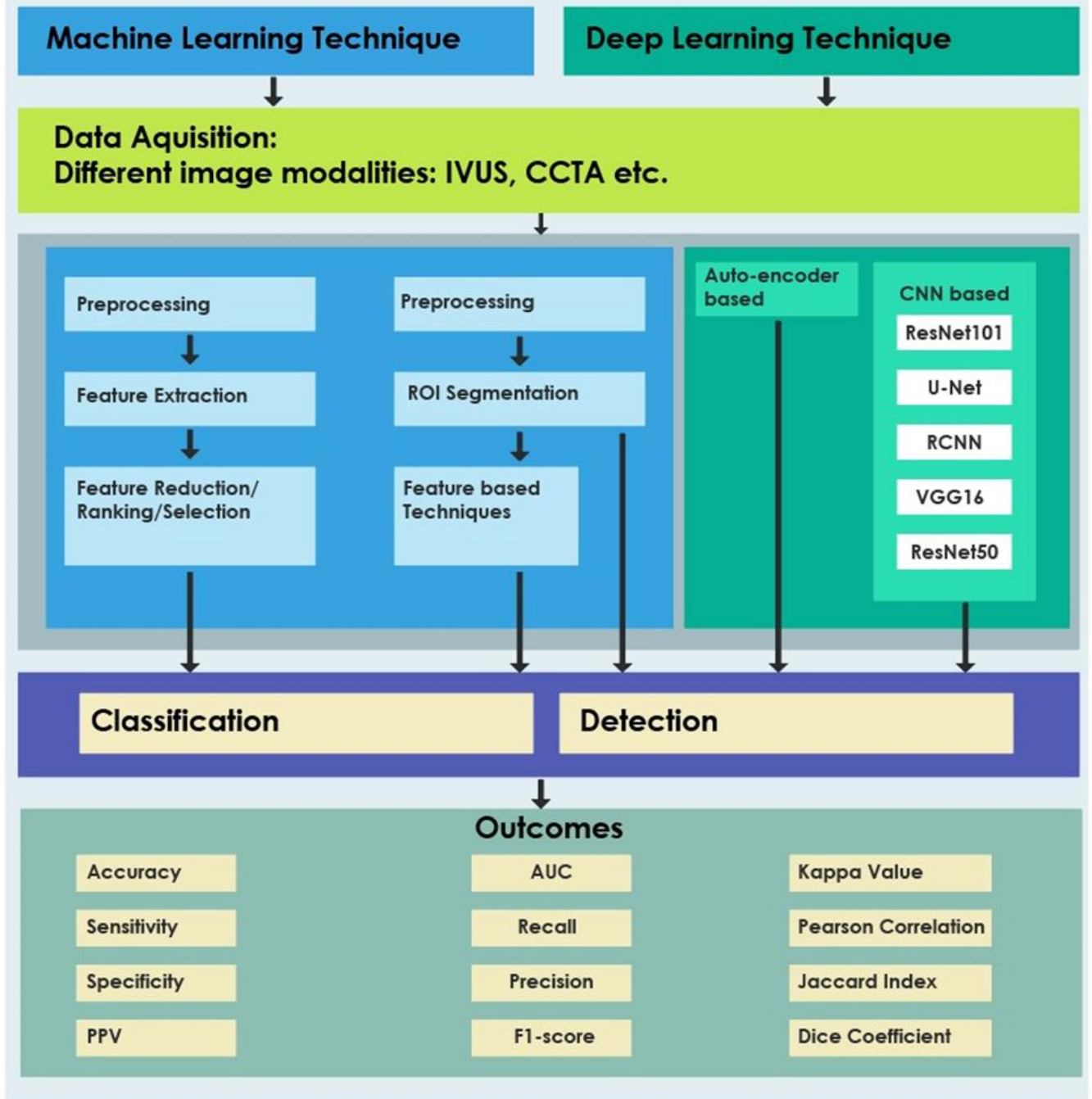

Figure 3. Processes, techniques and performance metrics of machine and deep learning models for coronary plaque characterization.

Deep Learning Techniques

Automated image recognition has improved tremendously since the widespread implementation of DL techniques, especially those with a convolutional neural network $(\mathrm{CNN})$ architecture. $\mathrm{CNN}$ is composed of a series of layers, in which discriminative image features are extracted from raw data automatically $[77,78]$. In general, DL recreates complex patterns from the assembly of simple ones. The input image is fed into the deep CNNs at initialization, and the data is propagated through layers generally consisting of convolution, pooling, rectified linear unit, and fully connected layer [79].

\subsubsection{Preprocessing/Segmentation}

Images obtained from different modalities may not be carry sufficient information for further processing or may be of low quality. Preprocessing helps to standardize image quality and increase image contrast. Denoising techniques include the anisotropic diffusion filter [80,81], Gaussian filter [82-84] and median filter. The adaptive histogram equalization technique can also be used to improve the image contrast. In order to remove interference, the Hough transform is used in [85]. The segmentation process is simplified by converting images to polar coordinates [86]. High intensity calcified plaques may also be normalized. Furthermore, Otsu's thresholding method is applied [87]. In [88], the multilevel discrete 
wavelet frame decomposition is used for image segmentation. In [11], a hybrid fuzzy $\mathrm{C}$-means and $\mathrm{k}$ nearest neighbor (HFCM-kNN) model is used for image segmentation. Other techniques include a region-growing method along with thresholding [89], and deformable models used for ROI extraction. The border can be delineated using k-means clustering and the Rayleigh mixture model [90]. Moreover, shadowed regions surrounding the plaque can be detected and removed using dynamic programming [91].

\subsubsection{Feature Extraction}

To accurately detect the plaque, certain features are extracted from the image. Using the widely used gray level co-occurrence matrix (GLCM), textural features can be extracted based on the spatial relationship between pixels [92-94]. Haralick's textural features exhibited a good discrimination for arterial wall and lumen identification [70]. In [95], the Gabor transform for different scales and angles along with six measurements of entropy were explored. Likewise, first order statistics (FOS) can be used for texture analysis [96]. To extract coronary lumen and plaque features, an adjacent pattern method was implemented. In addition, features are obtained using color moments of the histogram followed by statistical feature extraction [97]. Other authors have used an attenuation coefficient, which extracts features by selecting a window for each pixel so as to mark the pixular labels based on plaque type [84]. Different textural patterns exist in various coronary plaque components. Textural analysis methods such as neighboring gray level (NGL), local binary pattern (LBP) and modified run length (MRL) are suitable for distinguishing such plaque patterns. In order to differentiate the dense calcium tissues more significantly, fractal dimensions of the plaque components are computed using the box counting method. In addition, gray level run length matrix (GLRLM) and Law's texture energy (LTE) are used to analyze the textural patterns in plaques. The various plaque components can be extracted using a multiresolution decomposition method, i.e., fast wavelet transform (FWT), by obtaining the frequency content of the images [98]. Generally, the plaque components are characterized by different intensity levels. Specifically, dense calcium is associated with the highest intensity components. Extracting intensity of the plaque components improves the classification accuracy. Properties such as individual calcification levels, image brightness, and contrast, are obtained using an intensity histogram (IH). The neighborhood gray tone difference matrix (NGTDM), gray level difference statistics (GLDS), and invariant moment (IM) are incorporated to extract plaque textural features [99]. In [73], a multi-class coronary plaque detection framework random radius symmetry (RRS) containing contextual features of plaque was proposed that supplemented the training data of coronary plaques. Likewise, local indicators of spatial association (LISA) and run length (RL) are used to describe the textural features by detection. Several other algorithms have also been proposed for feature extraction, such as open lumen border tracing (OLBT), closed lumen border tracing (CLBT), extracting confluent components (ECC), and plaque burden assessment (PBA) [11].

\subsubsection{Feature Reduction/Selection/Ranking/Organization}

Several factors influence the success of classification algorithms in any given task. For instance, the degree of redundant information reduces the efficiency of plaque classification. In [88], a fuzzy complementary criterion (FuzCoC) was used to select the features for plaque component discrimination. To identify the best extracted features, the $t$-test or wrapped feature selection (WRP) [83] can be used. In [69], among feature selection techniques such as Relief-F, recursive feature elimination, and the Fisher method, the latter achieved the highest accuracy for ranking feature discriminative power. Further, principal component analysis (PCA) and genetic algorithms (GA) can be used to select the optimal feature set from the original feature set for tissue characterization. Informative features obtained using PCA often contain small values, which are normalized using the Z-score function [100]. In [95], feature reduction was performed using locality sensitive discriminant analysis (LSDA) and neighborhood preserving embedding (NPE), and the results were compared with no feature reduction on the dataset. The latter test generated 
the most discriminant features. Thereafter, the generated features were selected using the analysis of variance (ANOVA) statistic. The Wilcoxon signed-rank test can also be used for feature organization [101].

\subsubsection{Classification}

Plaques can be effectively categorized as calcified and non-calcified plaques using the Bayesian classifier [80]. In [92], an overall classification accuracy of $80.41 \%$ was achieved using the random forest (RF) classifier, in which the plaque was categorized into four types, namely calcium, lipid pool, fibrous tissue, and mixed plaques. Moreover, RF classifiers can classify the plaque into three classes, namely dense calcium (DC), necrotic core (NC), and fibrotic tissue (FT) and fibro-fatty tissue (FFT). Support vector machines (SVM) [73] are effectively used for coronary plaque characterization in most studies that utilize different kernel functions. SVM with a second order polynomial kernel function produced better performance parameters for carotid and coronary plaque characterization [99]. Error correcting output codes (ECOC) are the preferred medium for multiclass plaque classification [91]. For the discrimination of dense calcium tissue, the deep belief network model offers improved plaque characterization performance. Further, the atheromatous plaque can be characterized by a neuro fuzzy classifier. The FaIRLiC, hybrid ensemble classifier (feed forward neural network (FFNN), RF, adaptive boosting) can classify coronary plaque regions with high accuracy $[94,98]$.

For plaque classification, deep learning models such as ResNet50-v2 and DenseNet121 from ImageNet are used for binary as well as multiclass plaque classifications [102]. Plaques may be of varying severity. Based on their severity, they may be categorized as mild, moderate, or severe using a SVM-based CNN. Moreover, stenosis can be detected using U-Net and V-net [103]. Table 1 summarizes these various classification methodologies.

In [112], calcified regions are identified by detecting the acoustic shadow and analyzing the ROI extracted for the presence or absence of calcification in the IVUS images. The results of the proposed algorithm showed high correlation with human expert measurements. Likewise, a semiautomatic method for segmentation and quantification of calcified plaques was applied on IVOCT images based on a level-set model approach [87]. Along with quantified analysis of calcification, its boundary could be detected [113]. Detection of calcified plaque in the presence of acoustic shadowing is quite challenging. A Markov random field and graph searching algorithm was implemented in [90]. Along with calcified plaque detection, the lumen was detected using the K-means algorithm [82]. The calcified plaque could be determined based only on its $3 \mathrm{D}$ position, independent of the volume and shape of the plaque, using a blob enhancing filter [89]. However, coronary plaque composition, plaque position, and length of the plaque in abnormal coronary segments were better determined using the mean radial profile and SVM [69]. Soft and hard plaque detection can be ascertained using Fuzzy C-means (FCM) clustering, morphological processing, and a curve fitting function, which showed a high comparison coefficient compared with a manual plaque detection system. In addition to plaque detection, plaque shape and size can be quantitated [86]. Similarly, vulnerable plaques can be identified using a flexible neural tree (FNT) [81]. 
Table 1. Summary of various state-of-the-art techniques employed for plaque characterization using coronary artery scans.

\begin{tabular}{|c|c|c|c|c|c|c|c|c|}
\hline & Dataset & $\begin{array}{c}\text { Preprocessing/ROI } \\
\text { Segmentation }\end{array}$ & $\begin{array}{l}\text { Feature } \\
\text { Extraction }\end{array}$ & $\begin{array}{c}\text { Feature Reduc- } \\
\text { tion/Feature } \\
\text { Selection/ } \\
\text { Feature Rank- } \\
\text { ing/Organization }\end{array}$ & Detection & Classificatior & Task & Outcomes * \\
\hline [11] & $\begin{array}{l}599 \mathrm{VH}- \\
\text { IVUS } \\
\text { images } \\
\text { of 10 } \\
\text { patients }\end{array}$ & $\begin{array}{c}\text { Thresholding + } \\
\text { HFCM-kNN model }\end{array}$ & $\begin{array}{l}\text { CLBT }+ \\
\text { OLBT }\end{array}$ & & & $\begin{array}{l}\text { SVM } \\
\text { with } \\
\text { radial } \\
\text { basis } \\
\text { function } \\
\text { (RBF) }\end{array}$ & $\begin{array}{c}\text { Multiclass } \\
\text { (PIT, TCFA } \\
\text { and } \\
\text { CaTCFA) } \\
\text { and binary } \\
\text { (TCFA and } \\
\text { non-TCFA) }\end{array}$ & $\begin{array}{c}\text { For binary: } \\
\text { Pqacc.: } 81.03 \\
\text { Pq }_{\text {sen: }}: 84.81 \\
\text { Pq } \\
\text { Precec. }: 84.81 \\
\text { For multicn: } 84.81 \\
\text { Pqass } \\
\text { Pqavg.acc: } 98.42+0.01 \\
\text { Kappa: } 0.9198\end{array}$ \\
\hline [15] & $\begin{array}{l}\text { IVUS } \\
\text { images }\end{array}$ & & & & & $\begin{array}{l}\text { Neuro } \\
\text { Fuzzy }\end{array}$ & $\begin{array}{l}\text { Atheromatous } \\
\text { plaque } \\
\text { (fibrotic, } \\
\text { lipidic, } \\
\text { calcified, and } \\
\text { normal) }\end{array}$ & Pqavg.acc: 98.9 \\
\hline [21] & $\begin{array}{l}300 \\
\text { IVUS } \\
\text { frames } \\
\text { of } 10 \\
\text { patients }\end{array}$ & $\begin{array}{c}\text { Deformable } \\
\text { models }+ \\
\text { Estimation borders } \\
\text { by experts }\end{array}$ & $\begin{array}{c}\text { Co- } \\
\text { occurrence } \\
\text { matrix }+ \\
\text { LBP+ Mean } \\
\text { value + } \\
\text { Entropy + } \\
\text { Geometrical } \\
\text { features } \\
\end{array}$ & $t$-test & & $\mathrm{RF}$ & $\begin{array}{l}\text { Multiclass } \\
\text { (DC, NC, FT, } \\
\text { and FFT) }\end{array}$ & $\mathrm{Pq}_{\text {acc. }}: 85.65$ \\
\hline [22] & $\begin{array}{l}553 \\
\text { IVUS } \\
\text { frames } \\
\text { of eight } \\
\text { patients }\end{array}$ & $\begin{array}{c}\text { ROI Extraction + } \\
\text { Otsu's automatic } \\
\text { thresholding }+ \\
\text { Pathological tissue } \\
\text { detection }\end{array}$ & \multicolumn{3}{|c|}{ CNN } & & $\begin{array}{l}\text { Multiclass } \\
\text { (DC, NC, FT, } \\
\text { FFT, Media) }\end{array}$ & $\begin{array}{c}\text { Overall accuracy: } \\
\text { 93.5Pqacc.:DC: } \\
\text { 98.5NC: 88.6FT: } \\
\text { 91.1FFT: 90.0Media: } \\
\text { 99.4 }\end{array}$ \\
\hline [25] & $\begin{array}{l}\text { IVUS } \\
\text { images } \\
\text { from } 11 \\
\text { patients }\end{array}$ & $\begin{array}{l}\text { Manual } \\
\text { segmentation by } \\
\text { expert }\end{array}$ & $\begin{array}{c}\text { LBP + FOS } \\
+ \text { GLCM + } \\
\text { LEM + } \\
\text { Extended } \\
\text { GLRLM + } \\
\text { Intensity }\end{array}$ & \multicolumn{2}{|l|}{ PCA } & $\mathrm{RF}$ & $\begin{array}{l}\text { Multiclass(DC, } \\
\text { NC, FT, and } \\
\text { FFT) }\end{array}$ & $\begin{array}{c}\text { AUC: } 0.845,0.704, \\
0.783 \\
\text { Pqacc.: } 85.1,71.9,77.2 \\
\text { Pq }_{\text {qsen: }}: 82,81.2,80.6 \\
\text { Pq } \\
\text { spec.: } 87.1,59.6, \\
75.9 \\
\text { (Respectively for } \\
\text { Net1: FT/FFT or } \\
\text { NC/DC } \\
\text { Net2: FT or FFT } \\
\text { Net3: NC or DC) }\end{array}$ \\
\hline [27] & $\begin{array}{l}1000 \\
\text { IVOCT } \\
\text { images } \\
\text { from } 47 \\
\text { patients }\end{array}$ & $\begin{array}{c}\text { Anisotropic } \\
\text { diffusion + Polar } \\
\text { Transformation+ } \\
\text { Hough Transform }\end{array}$ & $\begin{array}{c}\text { Intensity + } \\
\text { HOG + LBP } \\
+ \text { FV + } \\
k \text {-means } \\
\text { clustering }\end{array}$ & & & SVM & $\begin{array}{l}\text { Multiclass } \\
\text { (normal, } \\
\text { fibrous } \\
\text { plaque, fibro- } \\
\text { atheroma, } \\
\text { plaque } \\
\text { rupture, } \\
\text { fibro-calcific } \\
\text { plaque) }\end{array}$ & $\begin{array}{c}\text { Pqavg.acc.: } 90 \\
\text { With standard } \\
\text { deviation of } 0.02\end{array}$ \\
\hline [67] & $\begin{array}{l}770 \text { OCT } \\
\text { images } \\
\text { of } 5 \\
\text { patients }\end{array}$ & ROI Extraction & LBP+GLCM & \multirow{2}{*}{\multicolumn{2}{|c|}{ CNN (U Net) }} & & $\begin{array}{l}\text { Multiclass } \\
\text { (lipid tissue, } \\
\text { fibrous } \\
\text { tissue, } \\
\text { background) }\end{array}$ & Pqacc: 95.8 \\
\hline [70] & $\begin{array}{l}435 \\
\text { IVUS } \\
\text { images }\end{array}$ & $\begin{array}{c}\text { Polar } \\
\text { Transformation + } \\
\text { Gaussian filter } \\
\text { +Median filter+ } \\
\text { Anisotropic } \\
\text { Diffusion filter }\end{array}$ & $\begin{array}{l}\text { Haralick's } \\
\text { +Laws' } \\
\text { textural } \\
\text { feature }\end{array}$ & & & SVM & $\begin{array}{l}\text { Two class } \\
\text { (fibrotic } \\
\text { plaque and } \\
\text { normal) }\end{array}$ & $\begin{array}{c}\text { AUC: } 0.97 \\
\text { Jaccard Index: } 0.85\end{array}$ \\
\hline [72] & $\begin{array}{c}6556 \\
\text { OCT } \\
\text { images } \\
\text { from } 49 \\
\text { patients }\end{array}$ & $\begin{array}{l}\text { ROI+ Dynamic } \\
\text { programming + } \\
\text { Gaussian filter }\end{array}$ & $\begin{array}{l}\mathrm{CNN}+\text { Mor- } \\
\text { phological } \\
\text { features }\end{array}$ & $\begin{array}{l}\text { Wilcoxon signed } \\
\text { rank test }\end{array}$ & & RF & $\begin{array}{l}\text { Binary class: } \\
\text { (fibro-lipidic } \\
\text { and } \\
\text { fibrocalcific } \\
\text { plaque) }\end{array}$ & $\begin{array}{c}\text { Fibro-lipidic plaque: } \\
\text { Pq } \text { sen. }: 84.8 \\
\text { Pqspec: } 97.8 \\
\text { Fibro-calcific plaque: } \\
\text { Pq } \\
\text { Pqsen: } 91.4 \\
\text { Pq }: 95.7\end{array}$ \\
\hline
\end{tabular}


Table 1. Cont.

\begin{tabular}{|c|c|c|c|c|c|c|c|c|}
\hline & Dataset & $\underset{\text { Segmentation }}{\text { Preprocessing/ROI }}$ & $\begin{array}{l}\text { Feature } \\
\text { Extraction }\end{array}$ & $\begin{array}{l}\text { Feature Reduc- } \\
\text { tion/Feature } \\
\text { Selection/ } \\
\text { Feature Rank- } \\
\text { ing/Organization }\end{array}$ & Detection & Classification & Task & Outcomes * \\
\hline [73] & $\begin{array}{c}18 \text { CTA } \\
\text { images } \\
\text { Total: } \\
1786 \\
\text { cross } \\
\text { sections } \\
\text { with } \\
\text { Non } \\
\text { calcified } \\
\text { plaque } \\
\text { (NCP): } \\
729, \\
\text { Calcified } \\
\text { plaque } \\
\text { (CP): 511, } \\
\text { Mixed } \\
\text { plaques: } \\
546 .\end{array}$ & DRLSE & $\begin{array}{l}\text { RRS feature } \\
\text { vector }\end{array}$ & & & $\begin{array}{l}\text { SVM } \\
\text { (Gaus- } \\
\text { sian } \\
\text { kernel) }\end{array}$ & $\begin{array}{l}\text { Multiclass } \\
\text { (calcified, non } \\
\text { calcified and } \\
\text { mixed plaques) }\end{array}$ & $\begin{array}{c}\text { Average precision: } \\
92.6 \pm 1.9 \\
\text { Average recall: } \\
94.3 \pm 2.1\end{array}$ \\
\hline [80] & $\begin{array}{l}60 \text { IVUS } \\
\text { images } \\
\text { of } 7 \\
\text { patients }\end{array}$ & $\begin{array}{l}\text { Anisotropic } \\
\text { diffusion filter }+ \\
\text { Thresholding }\end{array}$ & & & $\begin{array}{l}\text { Deformable } \\
\text { models }\end{array}$ & Bayesian & $\begin{array}{c}\text { Two class } \\
\text { (calcified and } \\
\text { non-calcified } \\
\text { plaque) }\end{array}$ & $\begin{array}{l}\text { AUC: } 0.943 \\
\text { Pqspec: } 98.5 \\
\text { Pqsen: } 92.67\end{array}$ \\
\hline [83] & $\begin{array}{l}27 \text { OCT } \\
\text { pull- } \\
\text { backs of } \\
22 \\
\text { patients }\end{array}$ & $\begin{array}{c}\text { Gaussian filter + } \\
\text { Thresholding + } \\
k \text {-means }\end{array}$ & $\begin{array}{c}\mathrm{LBP}+ \\
\text { GLCM }\end{array}$ & WRP & & n & $\begin{array}{l}\text { Multiclass } \\
\text { (calcium, lipid } \\
\text { pool, fibrous } \\
\text { tissue, and } \\
\text { mixed Tissue) }\end{array}$ & $\begin{array}{c}\text { Pearson's } \\
\text { correlation } \\
\text { coefficient: } 0.97 \\
\text { (FT) }\end{array}$ \\
\hline [84] & $\begin{array}{l}\text { IVOCT } \\
\text { images } \\
\text { of } 11 \\
\text { patients }\end{array}$ & $\begin{array}{l}\text { Gaussian filter + } \\
\text { Otsu threshold } \\
\text { filtering }\end{array}$ & $\begin{array}{l}\text { Attenuation } \\
\text { coefficient + } \\
\text { GLCM }\end{array}$ & & & $\begin{array}{l}\text { SVM } \\
\text { (RBF) }\end{array}$ & $\begin{array}{l}\text { Multiclass } \\
\text { (fibrous, } \\
\text { calcification } \\
\text { and lipid } \\
\text { tissue) }\end{array}$ & Pqacc. $: 83$ \\
\hline [88] & $\begin{array}{l}\text { IVUS } \\
\text { images } \\
\text { of } 7 \\
\text { patients }\end{array}$ & $\begin{array}{l}\text { Multilevel discrete } \\
\text { wavelet frame } \\
\text { decomposition }\end{array}$ & $\begin{array}{c}\text { FOS + } \\
\text { GLCM + } \\
\text { LBP + RL + } \\
\text { Wavelet } \\
\text { Intensity } \\
\text { values }\end{array}$ & FuzCoC & & $\begin{array}{l}\text { SVM } \\
\text { (RBF) }\end{array}$ & $\begin{array}{l}\text { Multiclass } \\
\text { (calcium, } \\
\text { necrotic core, } \\
\text { fibrous, and } \\
\text { fibro-fatty) }\end{array}$ & Pqavg.acc: 81 \\
\hline [91] & $\begin{array}{c}\text { In-vivo } \\
\text { dataset: } \\
\text { VH- } \\
\text { IVUS } \\
2263 \\
\text { images } \\
\text { of } 10 \\
\text { patients } \\
\text { Ex-vivo } \\
\text { dataset:64 } \\
\text { images }\end{array}$ & $\begin{array}{l}\text { Shadow detection } \\
\text { using threshold }\end{array}$ & $\begin{array}{l}\mathrm{NGL}+\mathrm{LBP}+ \\
\mathrm{MRL}\end{array}$ & & & $\begin{array}{l}\text { SVM } \\
\text { and } \\
\text { ECOC }\end{array}$ & $\begin{array}{l}\text { Multiclass(calcium, } \\
\text { necrotic core, } \\
\text { and fibro fatty) }\end{array}$ & $\begin{array}{c}\text { Kappa values: } \\
0.639 \text { (in-vivo) and } \\
0.628 \text { (ex-vivo) }\end{array}$ \\
\hline [92] & $\begin{array}{l}50 \text { OCT } \\
\text { images } \\
\text { from } 3 \\
\text { patients }\end{array}$ & & $\begin{array}{c}\text { Co- } \\
\text { occurrence } \\
\text { matrix }+ \\
\text { LBP+ } \\
\text { Entropy }+ \\
\text { Mean value }\end{array}$ & & & $\mathrm{n}$ & $\begin{array}{l}\text { Multiclass } \\
\text { (calcium, lipid } \\
\text { pool, fibrous } \\
\text { tissue, and } \\
\text { mixed plaque) }\end{array}$ & $\mathrm{Pq}_{\text {acc. }}: 80.41$ \\
\hline [93] & $\begin{array}{l}300 \\
\text { IVUS } \\
\text { images } \\
\text { of } 7 \\
\text { patients }\end{array}$ & $\begin{array}{c}\text { Multilevel discrete } \\
\text { wavelet frames } \\
\text { decomposition + } \\
\text { SOFM }\end{array}$ & $\begin{array}{c}\text { FOS + } \\
\text { GLCM + RL } \\
+ \text { LBP + } \\
\text { wavelets + } \\
\text { LISA }\end{array}$ & & & FaIRLiC & $\begin{array}{l}\text { Multiclass (DC, } \\
\text { NC, FT, and } \\
\text { FFT) }\end{array}$ & $\begin{array}{c}\text { Testing accuracy: } \\
76.16 \%\end{array}$ \\
\hline [94] & $\begin{array}{l}\text { IVUS } \\
\text { images } \\
\text { of } 7 \\
\text { patients }\end{array}$ & $\begin{array}{l}\text { Border detection + } \\
\text { 2-D Kohonen's } \\
\text { self-organizing } \\
\text { feature map } \\
\text { (SOFM) }\end{array}$ & $\begin{array}{c}\text { FOS + } \\
\text { GLCM + WF } \\
+\mathrm{RL}+\mathrm{LBP}\end{array}$ & & & FaIRLiC & $\begin{array}{l}\text { Multiclass } \\
\text { (calcium, } \\
\text { necrotic core, } \\
\text { fibrous and } \\
\text { fibro lipid) }\end{array}$ & $\begin{array}{c}\text { Average } \\
\text { classification } \\
\text { Accuracy on each } \\
\text { frame: } 73.67\end{array}$ \\
\hline
\end{tabular}


Table 1. Cont

\begin{tabular}{|c|c|c|c|c|c|c|c|c|}
\hline & Dataset & $\underset{\text { Segmentation }}{\text { Preprocessing/ROI }}$ & $\begin{array}{c}\text { Feature } \\
\text { Extraction }\end{array}$ & $\begin{array}{c}\text { Feature Reduc- } \\
\text { tion/Feature } \\
\text { Selection/ } \\
\text { Feature Rank- } \\
\text { ing/Organization }\end{array}$ & Detection & Classification & Task & Outcomes * \\
\hline [95] & $\begin{array}{l}2646 \\
\text { Coro- } \\
\text { nary } \\
\text { Tomog- } \\
\text { raphy } \\
\text { Angiog- } \\
\text { raphy } \\
\text { (CTA) } \\
\text { images } \\
\text { of } 73 \\
\text { patients } \\
\text { (CP: } 28, \\
\text { NCP: } 15, \\
\text { Normal: } \\
\text { 30) }\end{array}$ & $\begin{array}{c}\text { Adaptive } \\
\text { Histogram } \\
\text { Equalization }\end{array}$ & $\begin{array}{l}\text { Gabor } \\
\text { Transform + } \\
\text { Entropy }\end{array}$ & ANOVA & & $\begin{array}{l}\text { SVM (RBF } \\
\text { and poly- } \\
\text { nomial } \\
\text { kernel) }\end{array}$ & $\begin{array}{l}\text { Multiclass } \\
\text { (normal, non } \\
\text { calcified and } \\
\text { calcified) }\end{array}$ & $\begin{array}{l}\text { Pqacc: }: 89.09 \\
\text { Pqppv: } 91.70 \\
\text { Pq } \\
\text { Pqsen. }: 91.83 \\
\text { Pq } 83.70\end{array}$ \\
\hline [96] & $\begin{array}{l}316 \\
\text { IVUS } \\
\text { images } \\
\text { of } 26 \\
\text { patients }\end{array}$ & $\begin{array}{l}\text { Thresholding + } \\
\text { Polar } \\
\text { transformation + } \\
\text { Morphological } \\
\text { operations }\end{array}$ & $\begin{array}{c}\text { FOS + FD } \\
\text { (Box } \\
\text { counting) + } \\
\text { GLCM + } \\
\text { GLRLM + } \\
\text { LTE }\end{array}$ & PCA & & $\begin{array}{c}\text { Deep } \\
\text { belief } \\
\text { network }\end{array}$ & $\begin{array}{l}\text { Multiclass } \\
\text { (DC, NC, FT, } \\
\text { and FFT) }\end{array}$ & 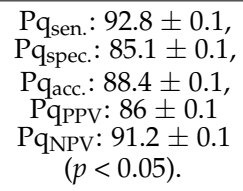 \\
\hline [97] & $\begin{array}{l}\text { IVUS } \\
\text { images } \\
\text { of } 7 \\
\text { patients }\end{array}$ & & $\begin{array}{c}\text { Adjacent } \\
\text { pattern } \\
\text { algorithm }+ \\
\text { Color } \\
\text { moments of } \\
\text { histogram + } \\
\text { Statistical } \\
\text { features }\end{array}$ & SVI & based CNN & & $\begin{array}{l}\text { Multiclass } \\
\text { (mild, } \\
\text { moderate } \\
\text { and severe) }\end{array}$ & 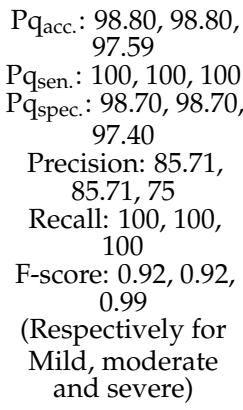 \\
\hline [98] & $\begin{array}{l}\text { IVUS } \\
\text { images } \\
\text { from } 11 \\
\text { patients }\end{array}$ & $\begin{array}{l}\text { Manual border } \\
\text { segmentation }\end{array}$ & $\begin{array}{c}\text { FOS + } \\
\text { GLCM + } \\
\text { GLRLM + } \\
\text { LBP + } \\
\text { Intensity + } \\
\text { Discrete } \\
\text { wavelet } \\
\text { features } \\
\text { +LTE }\end{array}$ & $\begin{array}{l}\text { Genetic } \\
\text { algorithm }\end{array}$ & & $\begin{array}{l}\text { Hybrid } \\
\text { ensemble } \\
\text { classi- } \\
\text { fier(FFNN+ } \\
\text { RF+ Ada } \\
\text { boost) }\end{array}$ & $\begin{array}{l}\text { Multiclass } \\
\text { (DC, NC, FT, } \\
\text { and FFT) }\end{array}$ & $\begin{array}{c}\text { Pqacc.: } 82.8,71.6,77 \\
\text { AUC: } 0.832,0.697, \\
0.787 \\
\text { Pq } \\
\text { sen. } 84.4,81.9, \\
74.9 \\
\text { Pqspec.: } 81.9,57.6, \\
82.4 \\
\text { PqPPV: } 71.2,72.4, \\
91.7 \\
\text { PqNPv: } 90.8,70.1, \\
55.9 \\
\text { (Respectively for } \\
\text { Net1: FT/FFT or } \\
\text { NC/DC } \\
\text { Net2: FT or FFT } \\
\text { Net3: NC or DC) }\end{array}$ \\
\hline [99] & $\begin{array}{c}2685 \\
\text { IVUS } \\
\text { images } \\
\text { of } 15 \\
\text { patients }\end{array}$ & ImgTracer software & $\begin{array}{c}\text { GLCM + } \\
\text { GLRLM + IH } \\
\text { + GLDS + } \\
\text { NGTDM + } \\
\text { IM + } \\
\text { Statistical } \\
\text { feature } \\
\text { matrix }\end{array}$ & & & $\begin{array}{l}\text { SVM (poly- } \\
\text { nomial } \\
\text { kernel 2nd } \\
\text { order) }\end{array}$ & $\begin{array}{l}\text { Coronary } \\
\text { and carotid } \\
\text { plaque }\end{array}$ & $\begin{array}{c}\text { Pqacc: }: 94.95 \\
\text { AUC: } 0.95 \\
\text { Pqsen. } 92.88 \\
\text { Pqspec: } 96.61 \\
\text { PqPPV: } 96.69\end{array}$ \\
\hline [100] & $\begin{array}{l}588 \mathrm{VH}- \\
\text { IVUS } \\
\text { images } \\
\text { of } 10 \\
\text { patients }\end{array}$ & $\begin{array}{c}\text { Fuzzy c means and } \\
k \text { means with } \\
\text { particle swarm } \\
\text { optimization }\end{array}$ & $\begin{array}{l}\mathrm{LBP}+ \\
\mathrm{GLCM}+ \\
\mathrm{MRL}\end{array}$ & PCA & & SVM (RBF) & $\begin{array}{l}\text { TCFA and } \\
\text { Non-TCFA }\end{array}$ & Pqacc.: 98.61 \\
\hline$[102]$ & $\begin{array}{c}4000 \\
\text { IVOCT } \\
\text { images } \\
\text { from } 49 \\
\text { patients }\end{array}$ & $\begin{array}{l}\text { Cartesian } \\
\text { Transformation }\end{array}$ & & $\begin{array}{r}\text { CNN from Im } \\
\text { ResNet50-v2 and D }\end{array}$ & $\begin{array}{l}\text { geNet } \\
\text { nseNet-121 }\end{array}$ & & $\begin{array}{l}\text { Binary class: } \\
\text { plaque } \\
\text { (calcified } \\
\text { plaque and } \\
\text { lipid/fibrous } \\
\text { plaque) and } \\
\text { no plaque }\end{array}$ & $\begin{array}{l}\text { Pqacc: }: 91.7 \\
P_{\text {sen. }}: 90.9 \\
\text { Pqspec: }: 92.4\end{array}$ \\
\hline
\end{tabular}


Table 1. Cont.

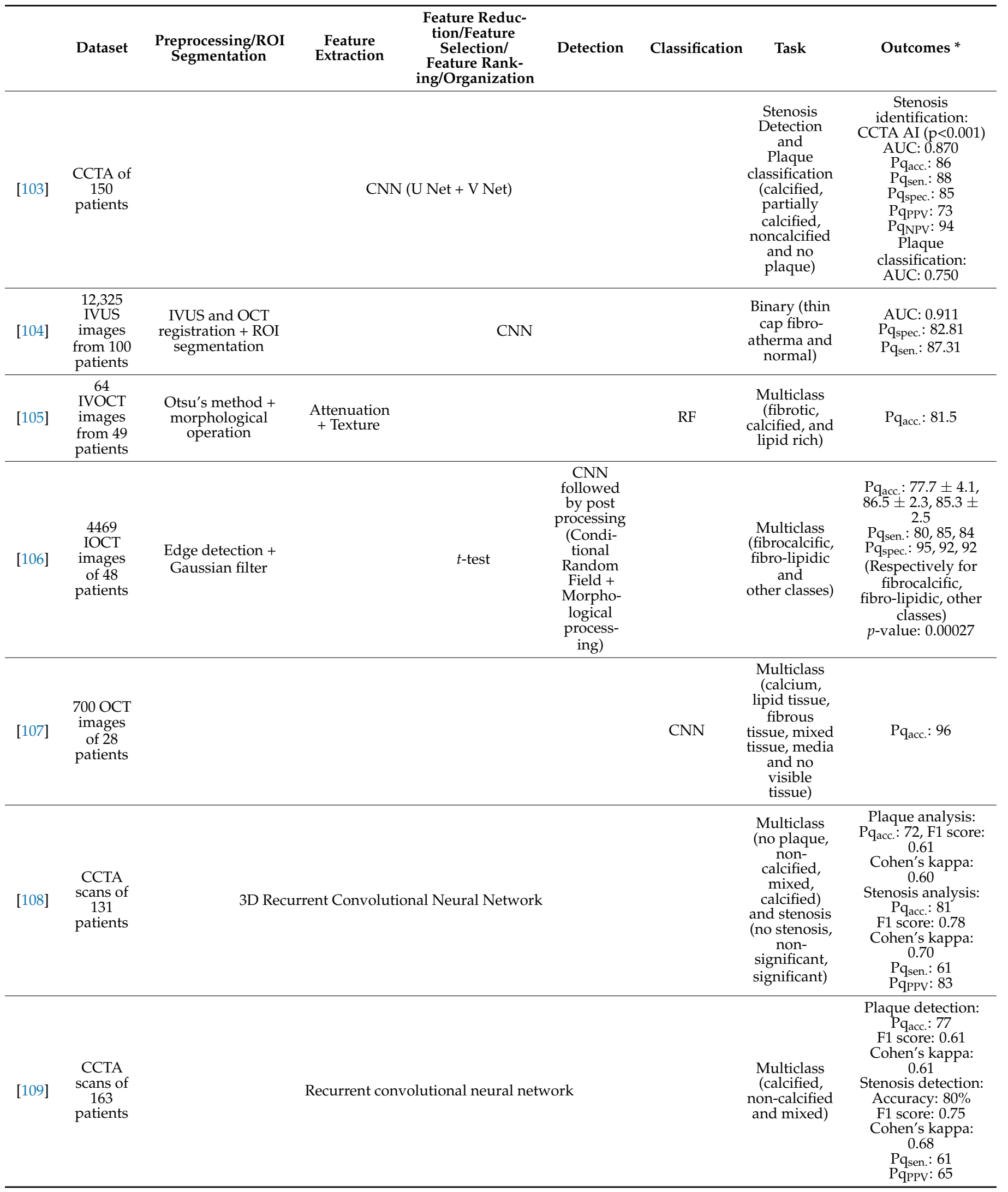


Table 1. Cont.

\begin{tabular}{|c|c|c|c|c|c|c|c|}
\hline & Dataset & $\underset{\text { Segmentation }}{\text { Preprocessing/ROI }}$ & $\begin{array}{l}\text { Feature } \\
\text { Extraction }\end{array}$ & $\begin{array}{c}\text { Feature Reduc- } \\
\text { tion/Feature } \\
\text { Selection/ } \\
\text { Feature Rank- } \\
\text { ing/Organization }\end{array}$ & Classification & Task & Outcomes * \\
\hline [110] & $\begin{array}{c}\text { CTA } \\
\text { scans } \\
\text { from } 25 \\
\text { patients }\end{array}$ & $\begin{array}{l}\text { Multiplanar } \\
\text { reformation } \\
\text { technique }\end{array}$ & & $\begin{array}{l}\text { 3D CNN U-Net } \\
\text { (Encoder-decoder) }\end{array}$ & & $\begin{array}{l}\text { Multiclass } \\
\text { (calcified } \\
\text { plaque, } \\
\text { non-calcified } \\
\text { plaque and } \\
\text { mixed } \\
\text { calcified } \\
\text { plaque) }\end{array}$ & $\begin{array}{c}\text { Dice scores: } 0.83 \text {, } \\
0.73,0.68 \\
\text { Pqsen: }: 85,76,72 \\
\text { PqpPv: } 82,69,62 \\
\text { Respectively for } \\
\text { CAP, NCAP, } \\
\text { MCAP }\end{array}$ \\
\hline [111] & $\begin{array}{l}2060 \\
\text { CTA } \\
\text { images } \\
\text { from } 60 \\
\text { patients }\end{array}$ & & $\begin{array}{l}\text { Higher- } \\
\text { order spectra } \\
\text { cumulants }\end{array}$ & $\begin{array}{l}\text { Multiple factor } \\
\text { analysis }+t \text {-test }\end{array}$ & SVM(RBF) & $\begin{array}{c}\text { Binary } \\
\text { (calcified, } \\
\text { noncalcified) }\end{array}$ & $\begin{array}{l}\text { Pqacc: }: 95.83 \\
\text { Pq } 194.54 \\
\text { Pqspec: }: 97.13 \\
\text { PqPpV: } 97.05\end{array}$ \\
\hline
\end{tabular}

* AUC: Area Under Curve, Pqppv (\%): Plaque Positive Predictive Value, PqNPV (\%): Plaque Negative Predictive Value, Pqsen. (\%): Plaque Sensitivity, $\mathrm{Pq}_{\text {spec. }}(\%)$ : Plaque Specificity, Pqacc. $(\%)$ : Plaque accuracy, $\mathrm{Pq}$ avg.acc. $(\%)$ : Plaque average accuracy, Precision $(\%)$.

CNN-based DL techniques are commonly used for plaque classification or identification. A distribution-preserving autoencoder-based neural network (DPAE-NN) implemented on IVOCT images was found to be best suited for characterization of heterogeneous plaques [114]. Images passed through the ResNet101 architecture with fc1000 as the output layer and a naïve Bayesian (NB) classifier outperformed other methods for calcified plaque detection [115]. In [116], high sensitivity was obtained for calcification detection using Inception-ResNet-v2 along with a naive Bayes classifier. Similarly, CNN architectures ResNet-50, ResNet-101 and Inception-v3 using SVM and the discriminant analysis (DA) classifier could characterize plaques more efficiently. In [101], the 3D CNN model, along with a SegNet architecture, was utilized for calcified plaque segmentation. In addition to plaque detection, the 3D U-Net CNN was used in challenging tasks such as coronary artery lumen delineation and stenosis grading [79]. The DL technique termed recurrent convolutional neural network ( $\mathrm{RCNN}$ ) detects coronary artery stenosis by extracting the coronary artery centerline during a skeletonization process [108,109]. Lumen and artery wall media detection, which is crucial in identifying plaque buildup in the walls of coronary vessels, can be achieved with a CNN (U-Net + VGG16 encoder) [71]. Coronary calcium assessment, or calcium score, is an important predictor of cardiovascular events. Calcium scores are predicted using a CNN (U-Net) in $[117,118]$. Table 2 summarizes the various detection methodologies described.

Table 2. Summary of various state-of-the-art techniques employed for plaque characterization using coronary artery scans.

\begin{tabular}{|c|c|c|c|c|c|c|c|c|}
\hline & Dataset & $\begin{array}{c}\text { Preprocessing/ROI } \\
\text { Segmentation }\end{array}$ & $\begin{array}{l}\text { Feature } \\
\text { Extraction }\end{array}$ & $\begin{array}{c}\text { Feature Reduc- } \\
\text { tion/Feature } \\
\text { Selection/ } \\
\text { Feature Rank- } \\
\text { ing/Organization }\end{array}$ & Detection & Classification & Task & Outcome * \\
\hline [2] & $\begin{array}{c}2175 \text { IVUS } \\
\text { images of } \\
10 \text { patients } \\
530 \text { images } \\
\text { with calcifi- } \\
\text { cation } \\
1645 \\
\text { images } \\
\text { without } \\
\text { calcifica- } \\
\text { tion }\end{array}$ & $\begin{array}{l}\text { Original image } \\
\text { resized and } \\
\text { converted to RGB }\end{array}$ & \multicolumn{3}{|c|}{ ResNet50, ResNet101, Inception-v3 } & $\begin{array}{l}\text { SVM and } \\
\text { DA }\end{array}$ & $\begin{array}{l}\text { Calcified } \\
\text { plaque } \\
\text { detection }\end{array}$ & $\begin{array}{l}\text { Pqacc: } 100 \\
\text { Pqqun: } 100 \\
\text { Pq quec: }_{100}\end{array}$ \\
\hline [66] & $\begin{array}{l}\text { CT images } \\
\text { of } 56 \\
\text { patients }\end{array}$ & Thresholding & \multicolumn{3}{|c|}{ CNN (ConvNet) } & & $\begin{array}{l}\text { Calcification } \\
\text { identifica- } \\
\quad \text { tion }\end{array}$ & $\begin{array}{c}\text { Pq } \\
\text { Pqsen: }: 91.24 \\
\text { Pqsec: } 95.37 \\
\text { PqPPV }: 90.5 \\
\text { Pearson coefficient: } \\
0.983 \\
\text { Cohen's kappa: } \\
0.879\end{array}$ \\
\hline
\end{tabular}


Table 2. Cont.

\begin{tabular}{|c|c|c|c|c|c|c|c|c|}
\hline & Dataset & $\begin{array}{c}\text { Preprocessing/ROI } \\
\text { Segmentation }\end{array}$ & $\begin{array}{l}\text { Feature } \\
\text { Extraction }\end{array}$ & $\begin{array}{c}\text { Feature Reduc- } \\
\text { tion/Feature } \\
\text { Selection/ } \\
\text { Feature Rank- } \\
\text { ing/Organization }\end{array}$ & Detection & Classification & Task & Outcome * \\
\hline [68] & $\begin{array}{l}\text { CCTA of } \\
493 \\
\text { patients }\end{array}$ & $\begin{array}{l}\text { Centerline } \\
\text { extraction }+ \\
\text { Clamping } \\
\text { technique }\end{array}$ & & & & $3 \mathrm{D} \mathrm{CNN}$ & $\begin{array}{l}\text { Atherosclerosis } \\
\text { detection }\end{array}$ & $\begin{array}{c}\text { Pqavg.acc: }: 90.9 \\
\text { PqPPV }: 58.8 \\
\text { s } \text { Pq }_{\text {sen. }}: 68.9 \\
\text { Pq } \\
\text { Pqpec: }: 93.6 \\
\text { PqNPV: } 96.1 \\
\text { Average AUC: } 0.91\end{array}$ \\
\hline [69] & $\begin{array}{l}32 \text { datasets } \\
\text { of CTA }\end{array}$ & $\begin{array}{l}\text { Normalization of } \\
\text { high intensity } \\
\text { calcified plaque }\end{array}$ & & Fisher method & $\begin{array}{l}\text { Mean } \\
\text { radial } \\
\text { profile }\end{array}$ & $\begin{array}{l}\text { SVM } \\
\text { Gaussian } \\
\text { RBF }\end{array}$ & $\begin{array}{c}\text { Soft } \\
\text { plaque } \\
\text { detection }\end{array}$ & $\begin{array}{c}\text { Pqacc.: } 88.4 \\
\text { Pq } q_{\text {sen. }}: 93.2 \\
\text { Pqsec: }: 80.3 \\
\text { Dice coefficient: } \\
0.832\end{array}$ \\
\hline [71] & $\begin{array}{l}435 \text { IVUS } \\
\text { scan } \\
\text { images }\end{array}$ & & $\mathrm{CNN}(\mathrm{U}$ & t + VGG16 encoder) & & & $\begin{array}{l}\text { Detection } \\
\text { of lumen } \\
\text { and media }\end{array}$ & $\begin{array}{c}\text { For media: } \\
\text { Avg Jaccard } \\
\text { measure: } 0.8085 \\
\text { Avg Dice score: } \\
0.8825 \\
\text { For Lumen: } \\
\text { Avg Jaccard } \\
\text { measure:0.7982 } \\
\text { Avg Dice } \\
\text { score::8846 }\end{array}$ \\
\hline [79] & $\begin{array}{l}78 \text { CCTA } \\
\text { images of } \\
18 \text { patients }\end{array}$ & & & U-Net CNN & & & $\begin{array}{l}\text { Coronary } \\
\text { artery } \\
\text { lumen seg- } \\
\text { mentation } \\
\text { (for } \\
\text { grading } \\
\text { stenosis) }\end{array}$ & Dice: 0.8291 \\
\hline [81] & $\begin{array}{l}1000 \text { OCT } \\
\text { images }\end{array}$ & $\begin{array}{c}\text { Polar } \\
\text { Transformation }+ \\
\text { Anisotropic } \\
\text { diffusion }\end{array}$ & & & $\begin{array}{l}\text { Flexible } \\
\text { neural tree }\end{array}$ & & $\begin{array}{l}\text { Vulnerable } \\
\text { plaque } \\
\text { detection }\end{array}$ & $\mathrm{Pq}_{\text {acc.: }}: 90.80$ \\
\hline [82] & $\begin{array}{l}27 \text { OCT } \\
\text { images } \\
\text { from } 10 \\
\text { patients }\end{array}$ & $\begin{array}{l}\text { Gaussian filter + } \\
\text { Thresholding }\end{array}$ & & & $\begin{array}{l}k \text {-means } \\
\text { clustering }\end{array}$ & & $\begin{array}{l}\text { Calcified } \\
\text { plaque } \\
\text { detection }\end{array}$ & $\begin{array}{c}\text { Pq }_{\text {sen. }}: 83 \\
\text { Pqpv }: 74 \\
\text { Pearson correlation: } \\
0.434\end{array}$ \\
\hline [85] & $\begin{array}{l}1000 \text { OCT } \\
\text { images }\end{array}$ & $\begin{array}{c}\text { Hough Transform + } \\
\text { Polar } \\
\text { transformation }\end{array}$ & & $\mathrm{CNN}$ & & & $\begin{array}{l}\text { Fibrous } \\
\text { plaque } \\
\text { detection }\end{array}$ & $\begin{array}{l}\text { Pqacc: } 94.12 \\
\text { Recall: } 94.12\end{array}$ \\
\hline [86] & $\begin{array}{l}60 \text { IVUS } \\
\text { images } \\
\text { from } 7 \\
\text { patients }\end{array}$ & $\begin{array}{c}\text { Polar } \\
\text { Transformation }\end{array}$ & & & $\begin{array}{c}\text { GLCM + } \\
\text { FCM + } \\
\text { ROI } \\
\text { selection + } \\
\text { Morpho- } \\
\text { logical } \\
\text { processing } \\
+ \text { Curve } \\
\text { fitting }\end{array}$ & & $\begin{array}{l}\text { Detection } \\
\text { (Hard } \\
\text { plaque and } \\
\text { soft } \\
\text { plaque) }\end{array}$ & $\begin{array}{l}\text { Pq }_{\text {spec. }}: 83 \\
\text { Pq }_{\text {sen }}: 91\end{array}$ \\
\hline [87] & $\begin{array}{l}106 \text { IOCT } \\
\text { images of } \\
8 \text { patients }\end{array}$ & $\begin{array}{c}\text { Otsu's } \\
\text { thresholding + } \\
\text { Edge detection }\end{array}$ & & & $\begin{array}{c}\text { Intensity + } \\
\text { level-set } \\
\text { model }\end{array}$ & & $\begin{array}{l}\text { Segmentation } \\
\text { of calcified } \\
\text { plaque }\end{array}$ & Pq acc. $: 78 \pm 9$ \\
\hline [89] & $\begin{array}{l}\text { CCTA of } 7 \\
\text { patients }\end{array}$ & $\begin{array}{l}\text { Thresholding }+3 \mathrm{D} \\
\text { region growing }\end{array}$ & & & $\begin{array}{l}\text { Blob } \\
\text { enhancing } \\
\text { filter }\end{array}$ & & $\begin{array}{l}\text { Stenosis by } \\
\text { Calcified } \\
\text { plaque }\end{array}$ & Precision:94.4 \\
\hline [90] & $\begin{array}{l}996 \text { in-vivo } \\
\text { IVUS } \\
\text { images of } \\
8 \text { patients }\end{array}$ & $\begin{array}{l}\text { Rayleigh mixture } \\
\text { model }\end{array}$ & & & $\begin{array}{l}\text { Markov } \\
\text { random } \\
\text { field and } \\
\text { Graph } \\
\text { searching } \\
\text { algorithm }\end{array}$ & & $\begin{array}{l}\text { Calcified } \\
\text { plaque } \\
\text { detection }\end{array}$ & $\begin{array}{l}\text { Pq } \\
\text { Pqsen: }: 94.68 \\
\text { Pqsec: } 95.82\end{array}$ \\
\hline [101] & $\begin{array}{l}8231 \text { IOCT } \\
\text { images of } \\
68 \text { patients }\end{array}$ & $\begin{array}{c}\text { Dynamic } \\
\text { programming + } \\
\text { semantic } \\
\text { segmentation } \\
\text { method + Gaussian } \\
\text { filter }\end{array}$ & & $\begin{array}{l}\text { Wilcoxon signed } \\
\text { rank test }\end{array}$ & $\underset{\text { SegNet }}{\text { 3D CNN }+}$ & & $\begin{array}{l}\text { Calcified } \\
\text { plaque seg- } \\
\text { mentation }\end{array}$ & $\begin{array}{l}\text { Pqsen.: } 86.2 \\
\text { Precision: } 75.8 \\
\text { F1 score: } 0.781\end{array}$ \\
\hline
\end{tabular}


Table 2. Cont.

\begin{tabular}{|c|c|c|c|c|c|c|c|}
\hline & Dataset & $\begin{array}{c}\text { Preprocessing/ROI } \\
\text { Segmentation }\end{array}$ & $\begin{array}{c}\text { Feature Reduc- } \\
\text { tion/Feature } \\
\text { Selection/ } \\
\text { Feature Rank- } \\
\text { ing/Organization }\end{array}$ & Detection & Classification & Task & Outcome * \\
\hline [112] & $\begin{array}{l}20 \text { IVUS } \\
\text { images }\end{array}$ & $\begin{array}{l}\text { Adaptive } \\
\text { thresholding }\end{array}$ & & $\begin{array}{l}\text { Priori infor- } \\
\text { mation of } \\
\text { the } \\
\text { acoustic } \\
\text { Shadow }\end{array}$ & & $\begin{array}{l}\text { Calcification } \\
\text { detection }\end{array}$ & $\begin{array}{l}\text { Pq } \text { spec. }_{\text {sp. }}: 88 \\
\text { Pq }: 84 \\
\text { AUC: } 0.87\end{array}$ \\
\hline [113] & $\begin{array}{l}2175 \text { IVUS } \\
\text { images of } \\
10 \text { patients }\end{array}$ & $\begin{array}{l}\text { Otsu thresholding }+ \\
\text { Morphological } \\
\text { operation }+ \\
\text { Empirical threshold }\end{array}$ & & & & $\begin{array}{l}\text { Detection } \\
\text { of calcifica- } \\
\text { tion } \\
\text { boundary }\end{array}$ & $\begin{array}{l}\text { Pqacc: }: 82 \\
\text { Pq }_{\text {sen: }}: 80 \\
\text { Pqspec: }: 84 \\
\text { PqPPV: } 83\end{array}$ \\
\hline [114] & $\begin{array}{l}30 \mathrm{OCT} \\
\text { images }\end{array}$ & & DPAE-NN & & & $\begin{array}{l}\text { Binary } \\
\text { (detection } \\
\text { of plaque } \\
\text { and normal } \\
\text { tissues) }\end{array}$ & $\begin{array}{c}\text { AUC: } 0.9132 \\
\text { Pqacc.: } 93.6 \\
\text { Kappa score: } 0.62\end{array}$ \\
\hline [115] & $\begin{array}{c}2175 \text { IVUS } \\
\text { image of } 10 \\
\text { patients } \\
\text { with } 530 \\
\text { calcified } \\
\text { images and } \\
1645 \\
\text { without cal- } \\
\text { cification }\end{array}$ & $\begin{array}{l}\text { Original image } \\
\text { resized and } \\
\text { converted into RGB }\end{array}$ & $\mathrm{CNN}$ architecture ResNet101 & & NB & $\begin{array}{l}\text { Calcification } \\
\text { detection }\end{array}$ & $\begin{array}{l}\text { Pqacc: }: 99.95 \\
\text { Pq } \\
\text { Pqsen: } 99.81 \\
\text { Pqspec: } 100 \\
\text { PqPPV: } 100 \\
\text { PqNPV: } 99.94\end{array}$ \\
\hline [116] & $\begin{array}{l}2175 \text { IVUS } \\
\text { images of } \\
10 \text { patients } \\
\text { Calcified } \\
\text { images: } \\
530 \\
\text { Noncalcified: } \\
1645\end{array}$ & $\begin{array}{l}\text { Images resized and } \\
\text { converted to RGB }\end{array}$ & & $\begin{array}{l}\text { Inception- } \\
\text { ResNet-v2 }\end{array}$ & NB & $\begin{array}{l}\text { Calcification } \\
\text { detection }\end{array}$ & $\begin{array}{l}\text { Pq } \\
\text { Pqsen: }: 100 \\
\text { Pqpec. } 95.87 \\
\text { PqpPr: } 88.63 \\
\text { Pqacc: } 96.87 \\
\text { AUC: } 0.9967\end{array}$ \\
\hline [117] & $\begin{array}{l}903 \mathrm{CT} \\
\text { scans }\end{array}$ & & ConvNet & & & $\begin{array}{l}\text { Calcium } \\
\text { scoring }\end{array}$ & $\begin{array}{c}\text { Cohen's kappa: } 0.95 \\
\text { Precision:77 } \\
\text { Recall: } 85 \\
\text { Pqacc.: } 99 \\
\text { Dice score: } 0.81\end{array}$ \\
\hline [118] & $\begin{array}{l}\text { CT scans of } \\
20084 \\
\text { individuals }\end{array}$ & & CNN (U-Net) & & & $\begin{array}{l}\text { Assessment } \\
\text { of calcium } \\
\text { score }\end{array}$ & AUC: 0.74 \\
\hline [119] & $\begin{array}{l}8914 \text { IVUS } \\
\text { images of } \\
80 \text { patients }\end{array}$ & $\mathrm{CNN}$ & & & & $\begin{array}{l}\text { Calcified } \\
\text { plaque } \\
\text { detection }\end{array}$ & $\begin{array}{c}\text { Average F1 score: } \\
0.67 \\
\text { Average precision: } \\
77 \\
\text { Average recall: } 83\end{array}$ \\
\hline [120] & $\begin{array}{l}105 \text { IVUS } \\
\text { pullback } \\
\text { dataset }\end{array}$ & $\begin{array}{c}\text { Polar } \\
\text { transformation }\end{array}$ & & & SVM (RBF) & $\begin{array}{l}\text { Calcified } \\
\text { plaque } \\
\text { detection }\end{array}$ & $\begin{array}{l}\text { Pqacc. }>90 \\
\text { Precision: } 96 \\
\text { Recall: } 93\end{array}$ \\
\hline [121] & $\begin{array}{l}713 \\
\text { grayscale } \\
\text { IVUS } \\
\text { images of } \\
18 \text { patients }\end{array}$ & & CNN U-Net architecture & & & 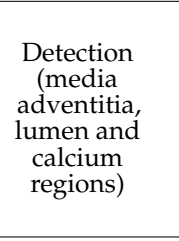 & $\begin{array}{c}\text { Average precision: } \\
73 \\
\text { Pqsen: } 72 \\
\text { Pqspec. } 99 \\
\text { Mean Dice score } \\
\text { function (DSC): } \\
\text { 0.67Spearman's } \\
\text { correlation: } 0.92\end{array}$ \\
\hline [122] & $\begin{array}{l}\text { CTA of } 12 \\
\text { patients }\end{array}$ & $\begin{array}{l}\text { Thresholding + } \\
\text { Difference of } \\
\text { Gaussian filter } \\
\text { (DOG) }\end{array}$ & & $\begin{array}{l}\text { Fuzzy c } \\
\text { means + } \\
\text { Median } \\
\text { filter }\end{array}$ & & $\begin{array}{l}\text { Calcified } \\
\text { plaque } \\
\text { detection }\end{array}$ & $\begin{array}{l}\text { Recall: } 94 \\
\text { Precision: } 94\end{array}$ \\
\hline [123] & $\begin{array}{l}\text { 2D axial } \\
\text { CTA } \\
\text { images of } \\
50 \text { patients }\end{array}$ & U-Net & & $\begin{array}{l}\text { CNN-RNN } \\
\text { model }\end{array}$ & $\mathrm{CNN}$ & $\begin{array}{l}\text { Segmentation } \\
\text { of coronary } \\
\text { artery } \\
\text { (stenosis } \\
\text { detection) }\end{array}$ & $\begin{array}{c}\text { Recall:95.9 } \\
\text { Precision: } 97.9 \\
\text { Pqacc. } 96.1\end{array}$ \\
\hline [124] & $\begin{array}{c}\text { CTA } \\
\text { images of } \\
30 \text { patients }\end{array}$ & $\begin{array}{c}\text { Hessian matrix+ } \\
\text { Thresholding+ } \\
\text { Morphological } \\
\text { operations }\end{array}$ & & & SVM & $\begin{array}{l}\text { Stenosis } \\
\text { detection }\end{array}$ & $\begin{array}{l}\text { Average Recall: } \\
\text { 94.08 } \\
\text { Precision: } 88.59\end{array}$ \\
\hline
\end{tabular}

${ }^{*}$ AUC: Area Under Curve, PqPPV (\%): Plaque Positive Predictive Value, $\mathrm{Pq}_{\mathrm{NPV}}(\%)$ : Plaque Negative Predictive Value, Pqsen. $(\%)$ : Plaque Sensitivity, Pq $q_{\text {spec. }}(\%)$ : Plaque Specificity, Pqacc. $(\%)$ : Plaque accuracy, Pqavg.acc. $(\%)$ : Plaque average accuracy, Precision (\%). 


\section{Discussion}

In this study, we present a systematic catalogue of CAD using ML and DL techniques for coronary atherosclerotic plaque characterization using various imaging modalities.

\subsection{Role of Various Modalities in Coronary Artery Disease}

When referenced to invasive CAG, CCTA has sensitivity of 73-99\%, specificity of $54-94 \%$, positive predictive value of $64-92 \%$ and negative predictive value (NPV) of $83-100 \%$ for coronary artery disease detection [36,125-131]. For detection of plaque in acute coronary syndrome, CCTA has good sensitivity and NPV (100\% and 100\%, respectively) [129]. Two meta-analyses, comprising 40 studies that compared CCTA with CAG and 41 diagnostic accuracy studies along with five prognostic studies, respectively, showed excellent pooled sensitivity of $99 \%$ and median NPV of 100\% [132]. Similarly, a meta-analysis of nine studies showed a high sensitivity of $96 \%$ (95\% CI: $93 \%$ to $98 \%$ ), specificity of $86 \%$ ( $95 \%$ CI: $83 \%$ to $89 \%$ ), positive likelihood ratio of 6.38 (95\% CI: 5.18 to 7.87 ) and negative likelihood ratio of 0.06 (95\% CI: 0.03 to 0.10 ) for CCTA against the reference standard CAG [28]. For detecting coronary artery disease in the low-to-intermediate risk group, the diagnostic accuracy improved with more advanced CT scanner models as well as with use of CT-based fractional flow reserve (FFR). CT FFR is derived using computational fluid dynamics simulation of the 3D anatomic coronary artery tree model, and is able to inform on the physiological significance of individual coronary artery stenoses. Using contrast gradient attenuation along an arterial lesion, CCTA has high diagnostic accuracy (ROC AUC of $0.88,95 \%$ CI $0.81-0.96, p<0.001$ ) and sensitivity, specificity, PPV and NPV of $77 \%$, $74 \%, 67 \%$ and $86 \%$, respectively, when referenced against invasive FFR-assessed coronary artery ischemia [133]. CT-based FFR showed a high specificity of $88 \%$ for coronary artery disease detection in [134]. Two meta-analyses involving studies that compared CCTA with invasive FFR showed good diagnostic accuracy of CCTA for the detection of functionally significant stenoses with pooled sensitivity of $92 \%$ and $84.6 \%$, and NPV of $84.6 \%$ and $87.3 \%$, respectively $[135,136]$. Detection of plaque characteristics through CCTA had a sensitivity and specificity greater than $90 \%$ compared to IVUS according to two meta-analyses involving 75 studies in total [137]. Detection of plaques with a napkin ring sign, which denote unstable plaque, by CCTA had a sensitivity of $93.8 \%$ and NPV of $66.7 \%$ when referenced against invasive IVUS findings [129]. Overall, the literature supports the use of CCTA for noninvasive diagnostic assessment of coronary artery disease.

\subsection{Role of $C A D$ in Coronary Artery Disease}

Automatic coronary plaque detection has been studied in 61 manuscripts included in this review report: 16 manuscripts are based on CCTA, 28 based on IVUS, 16 manuscripts are based on IVOCT, and one is based on X-ray angiography.

Most studies have implemented classification techniques to categorize image data sets as either containing plaque, no plaque, or mixed plaque. Different classifiers were used and the performance metrics of the classifiers reported in terms of outcomes, such as sensitivity, specificity, AUC, accuracy, PPV, and NPV. The majority of articles reported sensitivity (30 manuscripts), specificity (24 manuscripts) and accuracy (38 manuscripts) as performance metrics. Performance parameters such as AUC, PPV, and average precision were also used to assess performance. Among the classifiers, SVM was found to outperform, and was implemented in 14 articles [120,124]. The RF classifier [105] and FaIRLiC classifier were used in some studies. A novel approach using a hybrid classifier containing FFNN, $\mathrm{RF}$, and AdaBoost was implemented by some authors. Some works focused on plaque detection, i.e., to determine the presence or absence of plaque. $\mathrm{CNN}$ was the most preferred model for plaque detection (implemented in 23 studies) [104,106,107,110,119,121,123].

In this review, performance outcomes for plaque detection and classification using ML and DL were compared. In [90], the highest sensitivity of $94.68 \%$ was reported for detection of calcified plaque with acoustic shadowing using a Markov random field and graph searching algorithm, based on the evaluation of 996 in vivo IVUS images acquired 
from eight patients. Compared with IVOCT, CCTA showed a high sensitivity of $93.2 \%$ when tested on 32 datasets [69]. A high specificity of $98.5 \%$ was achieved for automated segmentation of calcified plaque using a Bayesian classifier for a dataset that comprised 60 IVUS images from seven patients [80]. IVUS outperformed other modalities with an average accuracy of $98.9 \%$ for the classification of atheromatous plaque, which confirmed its clinical utility in plaque characterization [15]. Furthermore, a PPV of $96.69 \%$ was achieved in coronary and carotid plaque characterization using the SVM classifier implemented on 2685 IVUS images obtained from 15 patients [99]. 100\% sensitivity, specificity, and accuracy were obtained using a DL approach for detecting calcification in IVUS images in a dataset that comprised 2175 images acquired from ten patients. The results were obtained using the ResNet-50, ResNet-101 and Inception-v3 CNN architecture with SVM classifier and discriminant analysis classifier [2]. Based on the aforementioned findings, IVUS outperforms the IVOCT and CCTA. CCTA demonstrated better results when compared with IVOCT, with an accuracy of $99 \%$ using CNN [117]. Studies showed that, by using the CNN ResNet101 architecture for feature extraction and a naïve Bayesian classifier, a PPV of $100 \%$, accuracy of $99.95 \%$, sensitivity of $99.81 \%$, and specificity $100 \%$ were achieved in for detecting calcification for IVUS images acquired from ten patients [115]. ImageNet using models ResNet50-v2 and DenseNet121 was used for binary and multiclass plaque classification in the dataset comprising 4000 images acquired from 49 patients, in which accuracy of $91.7 \%$, specificity of $92.4 \%$ and sensitivity of $90.9 \%$ were reported [102].

Supervised learning and a DL algorithm were combined to form a hybrid method SVM-based CNN algorithm, which effectively characterized the image pixels into plaque and non-plaque regions. This hybrid model achieved an accuracy of $98.8 \%$, sensitivity of $100 \%$, and specificity of $98.70 \%$ on IVUS images acquired from seven patients [97]. Similarly, CNN and RF were implemented on the IVOCT images of 49 patients for binary plaque classification, yielding classification sensitivities of $84.8 \%$ and $91.4 \%$, and specificities of $97.8 \%$ and $95.7 \%$, respectively, for fibro-lipidic and fibrocalcific plaques [72]. A hybrid novel image-based classification with an ensemble classifier consisting of FFNN, $\mathrm{RF}$ and AdaBoost showed an increased accuracy of $82.8 \%$ compared with other single classifiers [98]. Figure 4 depicts the performance of all the methods.

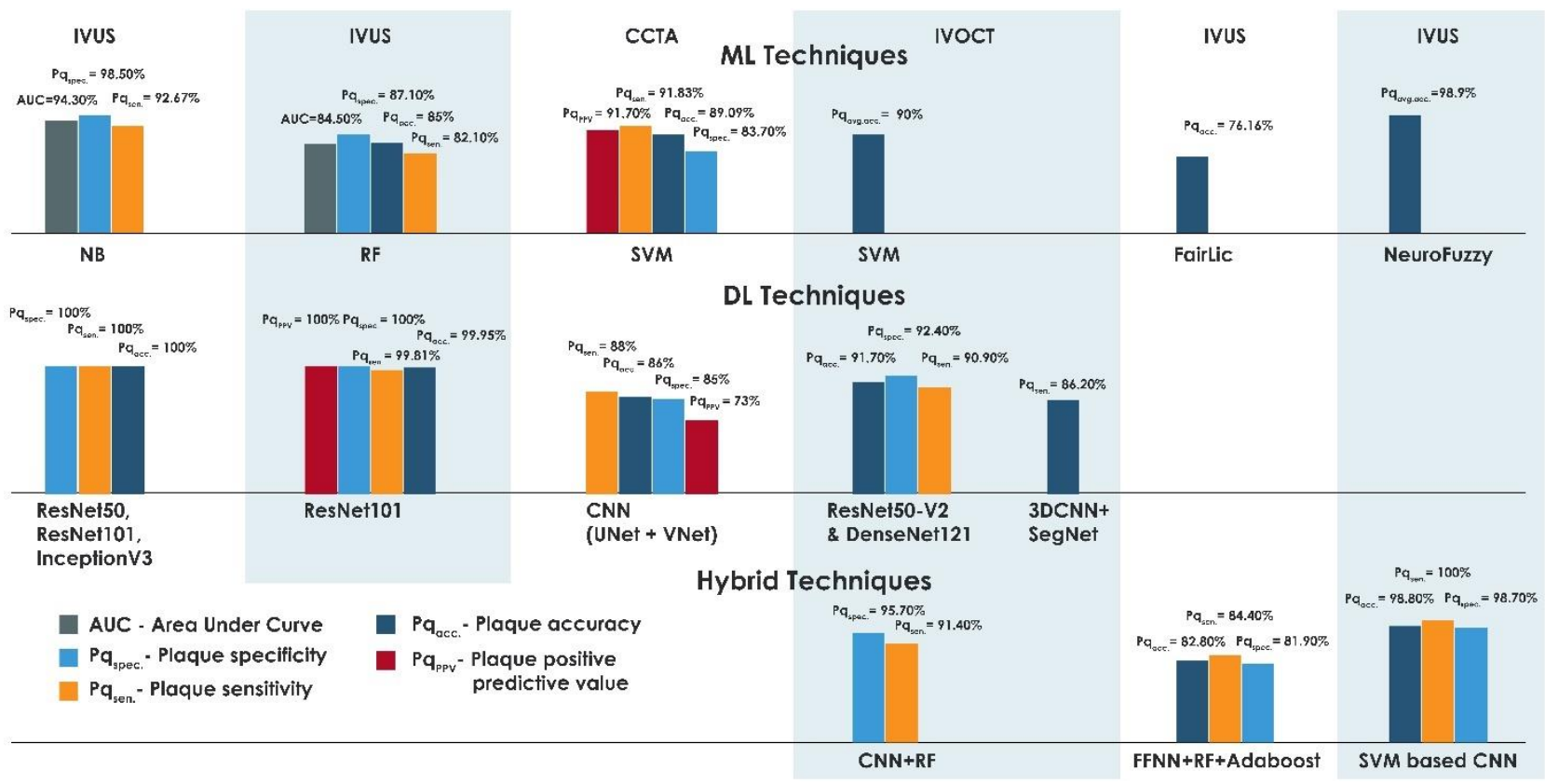

Figure 4. Plot of best-performing techniques with various modalities. 


\subsection{Research Opportunities and Future Direction}

It is observed that many studies in this field are based on IVUS. While invasive techniques are the reference standards for identifying and grading plaques and are helpful for understanding the underlying etiology of cerebrovascular disease, CCTA has emerged as the dominant noninvasive modality for studying coronary atherosclerosis. With CCTA, the identification of vulnerable plaques and their quantification relies on expert interpretation and is dependent on the degree of the interobserver variability. In addition, CCTA cannot supplant invasive techniques for more subtle plaque characteristics such as erosion and neovascularization [31]. While 3D reconstruction of plaque using CT slices would alert the physician to the presence of coronary artery disease at an early stage, it is advantageous that the investigator concentrates on hybrid feature extraction techniques when characterizing plaques using CAD based on CCTA images. There are challenges involved in the development of efficient CAD to characterize plaque [138-140]. These are discussed in detail below.

Availability of plaque datasets: Currently there is a lack of datasets that are publicly available. Most of the CAD are developed based on private datasets with low sample numbers. Hence, it is difficult to generalize from such analyses. Another challenge is to develop a large dataset with annotated plaque severity. This process is tedious and expensive, as it requires the input of expert radiologists. Data imbalance with various imaging modalities and their levels of severity may cause overfit or underfit of the training models. In order to handle skewed data, augmentation can be utilized in all modalities. The collection of more data of the different modalities can be achieved with the help of collaborative partner hospitals and clinics.

Comparative study on CAD using various modalities: There is a dearth of studies that have compared different modalities for the development of CAD to characterize plaques. Hence, a major challenge for researchers is to develop an efficient CAD to identify plaques using various imaging modalities, such as CCTA and IVUS images of the same patient. Investigators should concentrate on obtaining an automatic grading system using noninvasive imaging technique such as CCTA. This should then be compared with the expert grading system to produce a generalized CAD for plaque categorization. Finally, the CAD should be fast, noninvasive, inexpensive, and accurate, so that it can be used anywhere to obtain plaque deposition results without the need for expert involvement.

$C A D$ in the assessment of prognosis: Though several CAD are developed to detect and characterize plaques, none of the studies have reported on prognosis as well as responsiveness to treatment. Hybrid methods, such as a combination of ML and DL based approaches, can be further explored to develop CAD that correlates initial plaque deposition before and after treatment, as well as with long-term clinical outcome.

Generally, physicians or radiologists assess plaque deposition and its grading by using predefined image frames on IVUS, IVOCT and CCTA, and the results may vary from expert to expert based on their expertise in the various modalities, hence delaying treatment. Therefore, the diagnostic assessment of patients is typically performed by tertiary hospitals that are equipped to obtain and interpret the required images. It is thus difficult for geographically separated patients to obtain high-level diagnosis and treatment in a timely manner. In the future, cloud-based wireless healthcare systems can provide improved diagnostics (please refer to Figure 5). These consist of cloud-based methodologies, wherein hybrid CAD are available online to process the data. The CT scanner to obtain heart CT images noninvasively is connected to the cloud through a wireless network. Finally, CT images are processed and results sent to doctors and patients via mobile phone. This enables remote coordination of care by doctors of patients suspected of having coronary artery disease. This will assist in obtaining an overall goal of providing inexpensive yet high quality diagnostics in the rural areas of developing countries. 


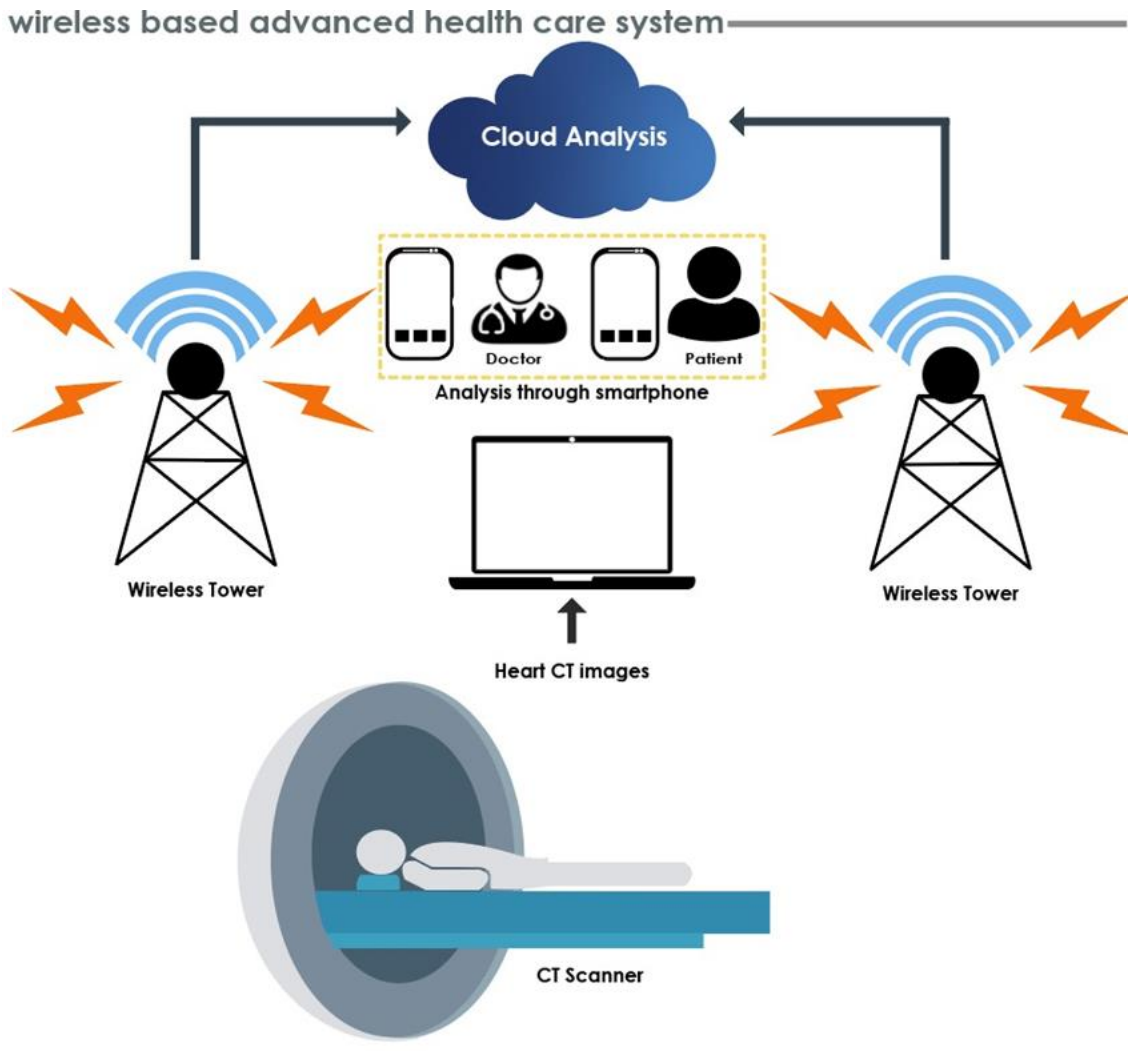

Figure 5. Future healthcare system using wireless networks.

\subsection{Limitations of the Study}

Some limitations of the study are given below:

1. The present review has been carried out based on manuscripts written in English. Other language manuscripts were not included during the review process;

2. The current review process included a plaque grading system using various modalities, and analysis of various $\mathrm{AI}$ algorithms to develop CAD for plaque categorization. However, review on grading during plaque deposition and after treatment was not given substantial consideration;

3. The specific reasons to select the algorithms based on AI were not mentioned. It was also unclear whether the proposed CAD can improve the survival of patients.

\section{Conclusions}

This review summarizes both medical and technical manuscripts for plaque characterization. A total of 61 medical manuscripts were used to understand the manual plaque grading schemes with various modalities and 61 technical papers were analyzed to understand the application of AI algorithms in the prediction of plaque deposition, classification, and detection. These methods were summarized and discussed along with various aspects of research challenges. Experimental results show that AI algorithms using ML and DL based methods have merits for identifying plaques, and can be used as a valuable resource in the medical decision-making process. In the future, these AI methods can be exploited to achieve better results by addressing future challenges and implementing the AI models in real clinical trials.

Author Contributions: Conceptualization, A.G., U.R. and U.R.A.; Methodology, A.G. and U.R.; Validation and review, A.A.J. and M.N.H.; Review and editing, E.J.C., R.-S.T., U.R.A. and P.D.B.; Original draft preparation, A.G., U.R., J.S., S.N.; Visualization, U.R.A. All authors have read and agreed to the published version of the manuscript.

Funding: This research received no external funding. 
Institutional Review Board Statement: Not applicable.

Informed Consent Statement: Not applicable.

Data Availability Statement: Not applicable.

Acknowledgments: The authors would like to thank the Manipal Academy of Higher Education (MAHE) for providing the required facility to carry out this research.

Conflicts of Interest: The authors declare no conflict of interest.

\section{References}

1. Libby, P.; Ridker, P.M.; Maseri, A. Inflammation and atherosclerosis. Circulation 2002, 105, 1135-1143. [CrossRef] [PubMed]

2. Sofian, H.; Than, J.C.M.; Mohammad, S.; Noor, N.M. Calcification Detection of Coronary Artery Disease in Intravascular Ultrasound Image: Deep Feature Learning Approach. Int. J. Integr. Eng. 2018, 10. Available online: https://publisher.uthm.edu. my/ojs/index.php/ijie/article/view/3473 (accessed on 9 August 2021).

3. Packard, R.R.; Libby, P. Inflammation in atherosclerosis: From vascular biology to biomarker discovery and risk prediction. Clin. Chem. 2008, 54, 24-38. [CrossRef]

4. Ross, R. Mechanisms of disease-Atherosclerosis-An inflammatory disease. N. Engl. J. Med. 1999, 340, 115-126. [CrossRef] [PubMed]

5. Slager, C.J.; Wentzel, J.J.; Gijsen, F.J.H.; Thury, A.; Van der Wal, A.C.; Schaar, J.A.; Serruys, P.W. The role of shear stress in the destabilization of vulnerable plaques and related therapeutic implications. Nat. Clin. Pract. Cardiovasc. Med. 2005, 2, 456-464. [CrossRef] [PubMed]

6. Falk, E.; Shah, P.K.; Fuster, V. Coronary plaque disruption. Circulation 1995, 92, 657-671. [CrossRef]

7. Bentzon, J.F.; Otsuka, F.; Virmani, R.; Falk, E. Mechanisms of plaque formation and rupture. Circ. Res. 2014, 114, 1852-1866. [CrossRef] [PubMed]

8. Athanasiou, L.S.; Fotiadis, D.I.; Michalis, L.K. Atherosclerotic Plaque Characterization Methods Based on Coronary Imaging; Academic Press: Cambridge, MA, USA, 2017. [CrossRef]

9. Virmani, R.; Kolodgie, F.D.; Burke, A.P.; Farb, A.; Schwartz, S.M. Lessons from sudden coronary death: A comprehensive morphological classification scheme for atherosclerotic lesions. Arter. Thromb. Vasc. Biol. 2000, 20, 1262-1275. [CrossRef] [PubMed]

10. Stary, H.C.; Chandler, A.B.; Dinsmore, R.E.; Fuster, V.; Glagov, S.; Insull, W., Jr.; Rosenfeld, M.E.; Schwartz, C.J.; Wagner, W.D.; Wissler, R.W. A Definition of Advanced Types of Atherosclerotic Lesions and a Histological Classification of Atherosclerosis. A Report from the Committee on Vascular Lesions of the Council on Arteriosclerosis, American Heart Association. Circulation 1995, 92, 1355-1374. [CrossRef]

11. Rezaei, Z.; Selamat, A.; Taki, A.; Rahim, M.S.M.; Kadir, M.R.A. Automatic plaque segmentation based on hybrid fuzzy clustering and $\mathrm{k}$ nearest neighborhood using virtual histology intravascular ultrasound images. Appl. Soft Comput. 2017, 53, 380-395. [CrossRef]

12. Yabushita, H.; Bouma, B.E.; Houser, S.L.; Aretz, H.T.; Jang, I.-K.; Schlendorf, K.H.; Kauffman, C.R.; Shishkov, M.; Kang, D.-H.; Halpern, E.F.; et al. Characterization of Human Atherosclerosis by Optical Coherence Tomography. Circulation 2002, 106, 1640-1645. [CrossRef]

13. Jang, I.K.; Tearney, G.J.; MacNeill, B.; Takano, M.; Moselewski, F.; Iftima, N.; Shishkov, M.; Houser, S.; Aretz, H.T.; Halpern, E.F.; et al. In vivo characterization of coronary atherosclerotic plaque by use of optical coherence tomography. Circulation 2005, 111, 1551-1555. [CrossRef] [PubMed]

14. Kolossváry, M.; Szilveszter, B.; Merkely, B.; Maurovich-Horvat, P. Plaque imaging with CT-a comprehensive review on coronary CT angiography based risk assessment. Cardiovasc. Diagn. Ther. 2017, 7, 489-506. [CrossRef] [PubMed]

15. Selvathi, D.; Emimal, N.; Selvaraj, H. Automated characterization of Atheromatous plaque in intravascular ultrasound images using Neuro fuzzy classifier. Int. J. Electron. Telecommun. 2012, 58, 425-431. [CrossRef]

16. Liu, H.; Wingert, A.; Wang, J.; Zhang, J.; Wang, X.; Sun, J.; Chen, F.; Khalid, S.G.; Jiang, J.; Zheng, D. Extraction of Coronary Atherosclerotic Plaques from Computed Tomography Imaging: A Review of Recent Methods. Front. Cardiovasc. Med. 2021, 8, 597568. [CrossRef] [PubMed]

17. Moher, D.; Liberati, A.; Tetzlaff, J.; Altman, D.G.; The PRISMA Group. Preferred reporting items for systematic reviews and meta-analyses: The PRISMA statement. PLoS Med. 2009, 6, e1000097. [CrossRef]

18. Andrews, J.; Puri, R.; Kataoka, Y.; Nicholls, S.J.; Psaltis, P.J. Therapeutic modulation of the natural history of coronary atherosclerosis: Lessons learned from serial imaging studies. Cardiovasc. Diagn. Ther. 2016, 6, 282-303. [CrossRef]

19. Carlier, S.G.; Tanaka, K. Studying coronary plaque regression with IVUS: A critical review of recent studies. J. Interv. Cardiol. 2006, 19, 11-15. [CrossRef]

20. Voros, S. Can Computed Tomography Angiography of the Coronary Arteries Characterize Atherosclerotic Plaque Composition? Is the CAT (Scan) Out of the Bag? JACC Cardiovasc. Interv. 2008, 1, 183-185. [CrossRef]

21. Athanasiou, L.S.; Karvelis, P.S.; Tsakanikas, V.D.; Naka, K.K.; Michalis, L.K.; Bourantas, C.V.; Fotiadis, D.I. A novel Semiautomated atherosclerotic plaque characterization method using Grayscale intravascular ultrasound images: Comparison with virtual histology. IEEE Trans. Inf. Technol. Biomed. 2012, 16, 391-400. [CrossRef] 
22. Olender, M.L.; Athanasiou, L.S.; Michalis, L.K.; Fotiadis, D.I.; Edelman, E.R. A Domain Enriched Deep Learning Approach to Classify Atherosclerosis Using Intravascular Ultrasound Imaging. IEEE J. Sel. Top. Signal Process. 2020, 14, 1210-1220. [CrossRef]

23. Nair, A.; Margolis, M.P.; Kuban, B.D.; Vince, D.G. Automated coronary plaque characterisation with intravascular ultrasound backscatter: Ex vivo validation. EuroIntervention 2007, 3, 113-120. [PubMed]

24. Nair, A.; Kuban, B.D.; Tuzcu, E.M.; Schoenhagen, P.; Nissen, S.E.; Vince, D.G. Coronary plaque classification with intravascular ultrasound radiofrequency data analysis. Circulation 2002, 106, 2200-2206. [CrossRef] [PubMed]

25. Kim, G.Y.; Lee, J.H.; Na Hwang, Y.; Kim, S.M. A novel intensity-based multi-level classification approach for coronary plaque characterization in intravascular ultrasound images. Biomed. Eng. Online 2018, 17, 151. [CrossRef]

26. Taki, A.; Hetterich, H.; Roodaki, A.; Setarehdan, S.; Unal, G.; Rieber, J.; Navab, N.; König, A. A new approach for improving coronary plaque component analysis based on intravascular ultrasound images. Ultrasound Med. Biol. 2010, 36, 1245-1258. [CrossRef]

27. Xu, M.; Cheng, J.; Wong, D.W.K.; Taruya, A.; Tanaka, A.; Liu, J.; Foin, N.; Wong, P. Automatic image classification in intravascular optical coherence tomography images. In Proceedings of the 2016 IEEE Region 10 Conference (TENCON), Singapore, 22-25 November 2016; pp. 1544-1547. [CrossRef]

28. Gorenoi, V.; Schönermark, M.P.; Hagen, A. CT coronary angiography vs. invasive coronary angiography in CHD. GMS Health Technol. Assess. 2012, 8, Doc02. [CrossRef]

29. Albus, C.; Barkhausen, J.; Fleck, E.; Haasenritter, J.; Lindner, O.; Silber, S. The diagnosis of chronic coronary heart disease. Dtsch. Aerzteblatt Online 2017, 114, 712-719. [CrossRef] [PubMed]

30. Puchner, S.B.; Liu, T.; Mayrhofer, T.; Truong, Q.A.; Lee, H.; Fleg, J.L.; Nagurney, J.T.; Udelson, J.E.; Hoffmann, U.; Ferencik, M. High-risk plaque detected on coronary CT angiography predicts acute coronary syndromes independent of significant stenosis in acute chest pain: Results from the ROMICAT-II trial. J. Am. Coll. Cardiol. 2014, 64, 684-692. [CrossRef]

31. Murgia, A.; Balestrieri, A.; Crivelli, P.; Suri, J.S.; Conti, M.; Cademartiri, F.; Saba, L. Cardiac computed tomography radiomics: An emerging tool for the non-invasive assessment of coronary atherosclerosis. Cardiovasc. Diagn. Ther. 2020, 10, 2005-2017. [CrossRef]

32. Infante, T.; Forte, E.; Schiano, C.; Cavaliere, C.; Tedeschi, C.; Soricelli, A.; Salvatore, M.; Napoli, C. An integrated approach to coronary heart disease diagnosis and clinical management. Am. J. Transl. Res. 2017, 9, 3148-3166.

33. Doh, J.H.; Koo, B.K.; Nam, C.W.; Kim, J.H.; Min, J.K.; Nakazato, R.; Silalahi, T.; Prawira, H.; Choi, H.; Lee, S.Y.; et al. Diagnostic value of coronary $\mathrm{CT}$ angiography in comparison with invasive coronary angiography and intravascular ultrasound in patients with intermediate coronary artery stenosis: Results from the prospective multicentre FIGURE-OUT (Functional Imaging criteria for GUiding REview of invasive coronary Angiography, intravascular Ultrasound, and coronary computed Tomographic angiography) study. Eur. Heart J. Cardiovasc. Imaging 2014, 15, 870-877. [CrossRef]

34. Mowatt, G.; Cummins, E.; Waugh, N.; Walker, S.; Cook, J.; Jia, X.; Hillis, G.S.; Fraser, C. Systematic review of the clinical effectiveness and cost-effectiveness of 64-slice or higher computed tomography angiography as an alternative to invasive coronary angiography in the investigation of coronary artery disease. 2008. In NIHR Health Technology Assessment Programme: Executive Summaries; NIHR Journals Library: Southampton, UK, 2003.

35. Miller, J.M.; Rochitte, C.E.; Dewey, M.; Arbab-Zadeh, A.; Niinuma, H.; Gottlieb, I.; Paul, N.; Clouse, M.E.; Shapiro, E.P.; Hoe, J.; et al. Diagnostic performance of coronary angiography by 64-row CT. N. Engl. J. Med. 2008, 359, 2324-2336. [CrossRef]

36. Budoff, M.J.; Dowe, D.; Jollis, J.G.; Gitter, M.; Sutherland, J.; Halamert, E.; Scherer, M.; Bellinger, R.; Martin, A.; Benton, R.; et al. Diagnostic Performance of 64-Multidetector Row Coronary Computed Tomographic Angiography for Evaluation of Coronary Artery Stenosis in Individuals without Known Coronary Artery Disease: Results from the Prospective Multicenter ACCURACY (Assessment by Coronary Computed Tomographic Angiography of Individuals Undergoing Invasive Coronary Angiography) Trial. J. Am. Coll. Cardiol. 2008, 52, 1724-1732.

37. Meijboom, W.B.; Meijs, M.F.; Schuijf, J.; Cramer, M.J.; Mollet, N.R.; van Mieghem, C.A.; Nieman, K.; van Werkhoven, J.M.; Pundziute, G.; Weustink, A.C.; et al. Diagnostic Accuracy of 64-Slice Computed Tomography Coronary Angiography: A Prospective, Multicenter, Multivendor Study. J. Am. Coll. Cardiol. 2008, 52, 2135-2144. [CrossRef] [PubMed]

38. Khajouei, A.S.; Adibi, A.; Maghsodi, Z.; Nejati, M.; Behjati, M. Prognostic value of normal and nonobstructive coronary artery disease based on CT angiography findings. A 12 month follow up study. J. Cardiovasc. Thorac. Res. 2019, 11, 318-321. [CrossRef] [PubMed]

39. Morita, H.; Fujimoto, S.; Kondo, T.; Arai, T.; Sekine, T.; Matsutani, H.; Sano, T.; Kondo, M.; Kodama, T.; Takase, S.; et al. Prevalence of computed tomographic angiography-verified high-risk plaques and significant luminal stenosis in patients with zero coronary calcium score. Int. J. Cardiol. 2012, 158, 272-278. [CrossRef]

40. Iwasaki, K.; Matsumoto, T.; Aono, H.; Furukawa, H.; Samukawa, M. Prevalence of non-calcified coronary plaque on 64slice computed tomography in asymptomatic patients with zero and low coronary artery calcium. Can. J. Cardiol. 2010, 26, 377-380. [CrossRef]

41. Uretsky, S.; Rozanski, A.; Singh, P.; Supariwala, A.; Atluri, P.; Bangalore, S.; Pappas, T.; Fisher, E.A.; Peters, M.R. The presence, characterization and prognosis of coronary plaques among patients with zero coronary calcium scores. Int. J. Cardiovasc. Imaging 2011, 27, 805-812. [CrossRef]

42. Becker, C.R.; Nikolaou, K.; Muders, M.; Babaryka, G.; Crispin, A.; Schoepf, U.J.; Loehrs, U.; Reiser, M.F. Ex vivo coronary atherosclerotic plaque characterization with multi-detector-row CT. Eur. Radiol. 2003, 13, 2094-2098. [CrossRef]

43. Kelly, J.L.; Thickman, D.; Abramson, S.D.; Chen, P.R.; Smazal, S.F.; Fleishman, M.J.; Lingam, S.C. Coronary CT angiography findings in patients without coronary calcification. Am. J. Roentgenol. 2008, 191, 50-55. [CrossRef] [PubMed] 
44. Tanaka, A.; Shimada, K.; Yoshida, K.; Jissyo, S.; Tanaka, H.; Sakamoto, M.; Matsuba, K.; Imanishi, T.; Akasaka, T.; Yoshikawa, J. Non-invasive assessment of plaque rupture by 64-slice multidetector computed tomography-Comparison with intravascular ultrasound. Circ. J. 2008, 72, 1276-1281. [CrossRef]

45. Kashiwagi, M.; Tanaka, A.; Kitabata, H.; Tsujioka, H.; Kataiwa, H.; Komukai, K.; Tanimoto, T.; Takemoto, K.; Takarada, S.; Kubo, T.; et al. Feasibility of noninvasive assessment of thin-cap fibroatheroma by ultidetector computed tomography. JACC Cardiovasc. Imaging 2009, 2, 1412-1419. [CrossRef] [PubMed]

46. Maurovich-Horvat, P.; Hoffmann, U.; Vorpahl, M.; Nakano, M.; Virmani, R.; Alkadhi, H. The napkin-ring sign: CT signature of high-risk coronary plaques? JACC Cardiovasc. Imaging 2010, 3, 440-444. [CrossRef] [PubMed]

47. Seifarth, H.; Schlett, C.L.; Nakano, M.; Otsuka, F.; Károlyi, M.; Liew, G.; Maurovich-Horvat, P.; Alkadhi, H.; Virmani, R.; Hoffmann, U. Histopathological correlates of the napkin-ring sign plaque in coronary CT angiography. Atherosclerosis 2012, 224, 90-96. [CrossRef]

48. Schroeder, S. Non-invasive evaluation of atherosclerosis with contrast enhanced 16 slice spiral computed tomography: Results of ex vivo investigations. Heart 2004, 90, 1471-1475. [CrossRef]

49. Kimura, S.; Yonetsu, T.; Suzuki, K.; Isobe, M.; Iesaka, Y.; Kakuta, T. Characterisation of non-calcified coronary plaque by 16slice multidetector computed tomography: Comparison with histopathological specimens obtained by directional coronary atherectomy. Int. J. Cardiovasc. Imaging 2011, 28, 1749-1762. [CrossRef] [PubMed]

50. Kwan, A.C.; Cater, G.; Vargas, J.; Bluemke, D.A. Beyond Coronary Stenosis: Coronary Computed Tomographic Angiography for the Assessment of Atherosclerotic Plaque Burden. Curr. Cardiovasc. Imaging Rep. 2013, 6, 89-101. [CrossRef] [PubMed]

51. Kopp, A.F.; Schroeder, S.; Baumbach, A.; Kuettner, A.; Georg, C.; Ohnesorge, B.; Heuschmid, M.; Kuzo, R.; Claussen, C.D. Non-invasive characterisation of coronary lesion morphology and composition by multislice CT: First results in comparison with intracoronary ultrasound. Eur. Radiol. 2001, 11, 1607-1611. [CrossRef]

52. Schroeder, S.; Kopp, A.F.; Baumbach, A.; Meisner, C.; Kuettner, A.; Georg, C.; Ohnesorge, B.; Herdeg, C.; Claussen, C.D.; Karsch, K.R. Noninvasive detection and evaluation of atherosclerotic coronary plaques with multislice computed tomography. J. Am. Coll. Cardiol. 2001, 37, 1430-1435. [CrossRef]

53. Leber, A.W.; Knez, A.; Becker, A.; Becker, C.; von Ziegler, F.; Nikolaou, K.; Rist, C.; Reiser, M.; White, C.; Steinbeck, G.; et al. Accuracy of multidetector spiral computed tomography in identifying and differentiating the composition of coronary atherosclerotic plaques: A comparative study with intracoronary ultrasound. J. Am. Coll. Cardiol. 2004, 43, 1241-1247. [CrossRef]

54. Voros, S.; Rinehart, S.; Qian, Z.; Joshi, P.; Vazquez, G.; Fischer, C.; Belur, P.; Hulten, E.; Villines, T.C. Coronary atherosclerosis imaging by coronary CT angiography: Current status, correlation with intravascular interrogation and meta-analysis. JACC Cardiovasc. Imaging 2011, 4, 537-554. [CrossRef]

55. Koh, J.E.W.; Raghavendra, U.; Gudigar, A.; Ping, O.C.; Molinari, F.; Mishra, S.; Mathavan, S.; Raman, R.; Acharya, U. A novel hybrid approach for automated detection of retinal detachment using ultrasound images. Comput. Biol. Med. 2020, 120, 103704. [CrossRef]

56. Pham, T.-H.; Raghavendra, U.; Koh, J.E.W.; Gudigar, A.; Chan, W.Y.; Hamid, M.T.R.; Rahmat, K.; Fadzli, F.; Ng, K.H.; Ooi, C.P.; et al. Development of breast papillary index for differentiation of benign and malignant lesions using ultrasound images. $J$. Ambient. Intell. Humaniz. Comput. 2020, 12, 2121-2129. [CrossRef]

57. Raghavendra, U.; Gudigar, A.; Ciaccio, E.J.; Ng, K.H.; Chan, W.Y.; Rahmat, K.; Acharya, U.R. 2DSM vs. FFDM: A computer aided diagnosis based comparative study for the early detection of breast cancer. Expert Syst. 2019, 38, e12474. [CrossRef]

58. Raghavendra, U.; Gudigar, A.; Bhandary, S.V.; Rao, T.N.; Ciaccio, E.J.; Acharya, U.R. A Two Layer Sparse Autoencoder for Glaucoma Identification with Fundus Images. J. Med. Syst. 2019, 43, 299. [CrossRef]

59. Gudigar, A.; Raghavendra, U.; Devasia, T.; Nayak, K.; Danish, S.M.; Kamath, G.; Samanth, J.; Pai, U.M.; Nayak, V.; Tan, R.S.; et al. Global weighted LBP based entropy features for the assessment of pulmonary hypertension. Pattern Recognit. Lett. 2019, 125, 35-41. [CrossRef]

60. Gudigar, A.; Raghavendra, U.; Ciaccio, E.J.; Arunkumar, N.; Abdulhay, E.; Acharya, U.R. Automated categorization of multiclass brain abnormalities using decomposition techniques with MRI images: A comparative study. IEEE Access 2019, 7, 28498-28509. [CrossRef]

61. Gudigar, A.; Raghavendra, U.; San, T.R.; Ciaccio, E.J.; Acharya, U.R. Application of multiresolution analysis for automated detection of brain abnormality using MR images: A comparative study. Future Gener. Comput. Syst. 2019, 90, 359-367. [CrossRef]

62. Molinari, F.; Raghavendra, U.; Gudigar, A.; Meiburger, K.M.; Acharya, U.R. An efficient data mining framework for the characterization of symptomatic and asymptomatic carotid plaque using bidimensional empirical mode decomposition technique. Med. Biol. Eng. Comput. 2018, 56, 1579-1593. [CrossRef]

63. Raghavendra, U.; Gudigar, A.; Maithri, M.; Gertych, A.; Meiburger, K.M.; Yeong, C.H.; Madla, C.; Kongmebhol, P.; Molinari, F.; $\mathrm{Ng}$, K.H.; et al. Optimized multi-level elongated quinary patterns for the assessment of thyroid nodules in ultrasound images. Comput. Biol. Med. 2018, 95, 55-62. [CrossRef]

64. Acharya, U.R.; Sree, S.V.; Krishnan, M.M.; Molinari, F.; Saba, L.; Ho, S.Y.; Ahuja, A.T.; Ho, S.C.; Nicolaides, A.; Suri, J.S. Atherosclerotic risk stratification strategy for carotid arteries using texture-based features. Ultrasound Med. Biol. 2012, 38, 899-915. [CrossRef]

65. Raghavendra, U.; Bhandary, S.; Gudigar, A.; Acharya, U.R. Novel expert system for glaucoma identification using non-parametric spatial envelope energy spectrum with fundus images. Biocybern. Biomed. Eng. 2018, 38, 170-180. [CrossRef] 
66. Santini, G.; Della Latta, D.; Martini, N.; Valvano, G.; Gori, A.; Ripoli, A.; Susini, C.L.; Landini, L.; Chiappino, D. An automatic deep learning approach for coronary artery calcium segmentation. In EMBEC E NBC 2017; Eskola, H., Väisänen, O., Viik, J., Hyttinen, J., Eds.; Springer: Singapore, 2016; Volume 65. [CrossRef]

67. Zhang, C.; Li, H.; Guo, X.; Molony, D.; Guo, X.; Samady, H.; Giddens, D.P.; Athanasiou, L.; Nie, R.; Cao, J.; et al. Convolution neural networks and support vector machines for automatic segmentation of intracoronary optical coherence tomography. Mol. Cell. Biomech. 2019, 16, 153-161. [CrossRef]

68. Candemir, S.; White, R.D.; Demirer, M.; Gupta, V.; Bigelow, M.T.; Prevedello, L.; Erdal, B.S. Automated coronary artery atherosclerosis detection and weakly supervised localization on coronary $\mathrm{CT}$ angiography with a deep 3-dimensional convolutional neural network. Comput. Med. Imaging Graph. 2020, 83, 101721. [CrossRef] [PubMed]

69. Jawaid, M.M.; Riaz, A.; Rajani, R.; Reyes-Aldasoro, C.C.; Slabaugh, G. Framework for detection and localization of coronary non-calcified plaques in cardiac CTA using mean radial profiles. Comput. Biol. Med. 2017, 89, 84-95. [CrossRef]

70. Vercio, L.L.; Orlando, J.I.; Del Fresno, M.; Larrabide, I. Assessment of image features for vessel wall segmentation in intravascular ultrasound images. Int. J. Comput. Assist. Radiol. Surg. 2016, 11, 1397-1407. [CrossRef]

71. Balakrishna, C.; Dadashzadeh, S.; Soltaninejad, S. Automatic detection of lumen and media in the IVUS images using U-Net with VGG16 Encoder. arXiv 2018, arXiv:1806.07554.

72. Lee, J.; Prabhu, D.; Kolluru, C.; Gharaibeh, Y.; Zimin, V.N.; Dallan, L.A.P.; Bezerra, H.G.; Wilson, D.L. Fully automated plaque characterization in intravascular OCT images using hybrid convolutional and lumen morphology features. Sci. Rep. 2020, 10, 2596. [CrossRef]

73. Zhao, F.; Wu, B.; Chen, F.; Cao, X.; Yi, H.; Hou, Y.; He, X.; Liang, J. An automatic multi-class coronary atherosclerosis plaque detection and classification framework. Med. Biol. Eng. Comput. 2019, 57, 245-257. [CrossRef]

74. Gudigar, A.; Raghavendra, U.; Hegde, A.; Kalyani, M.; Ciaccio, E.J.; Acharya, U.R. Brain pathology identification using computer aided diagnostic tool: A systematic review. Comput. Methods Programs Biomed. 2020, 187, 105205. [CrossRef] [PubMed]

75. Raghavendra, U.; Gudigar, A.; Rao, T.N.; Ciaccio, E.J.; Ng, E.Y.K.; Acharya, U.R. Computer-aided diagnosis for the identification of breast cancer using thermogram images: A comprehensive review. Infrared Phys. Technol. 2019, 102, 103041. [CrossRef]

76. Gudigar, A.; Raghavendra, U.; Hegde, A.; Menon, G.; Molinari, F.; Ciaccio, E.; Acharya, U.R. Automated Detection and Screening of Traumatic Brain Injury (TBI) Using Computed Tomography Images: A Comprehensive Review and Future Perspectives. Int. J. Environ. Res. Public Health 2021, 18, 6499. [CrossRef]

77. Raghavendra, U.; Bhat, N.S.; Gudigar, A.; Acharya, U.R. Automated system for the detection of thoracolumbar fracture using a CNN architecture. Future Gener. Comput. Syst. 2018, 85, 184-189. [CrossRef]

78. Raghavendra, U.; Fujita, H.; Bhandary, S.; Gudigar, A.; Tan, J.H.; Acharya, U.R. Deep convolution neural network for accurate diagnosis of glaucoma using digital fundus images. Inf. Sci. 2018, 441, 41-49. [CrossRef]

79. Huang, W.; Huang, L.; Lin, Z.; Huang, S.; Chi, Y.; Zhou, J.; Zhang, J.; Tan, R.-S.; Zhong, L. Coronary Artery Segmentation by Deep Learning Neural Networks on Computed Tomographic Coronary Angiographic Images. In Proceedings of the 2018 40th Annual International Conference of the IEEE Engineering in Medicine and Biology Society (EMBC), Honolulu, HI, USA, 18-21 July 2018; pp. 608-611. [CrossRef]

80. Taki, A.; Najafi, Z.; Roodaki, A.; Setarehdan, S.K.; Zoroofi, R.A.; König, A.; Navab, N. Automatic segmentation of calcified plaques and vessel borders in IVUS images. Int. J. Comput. Assist. Radiol. Surg. 2008, 3, 347-354. [CrossRef]

81. Tian, W.; Pang, Y.; Niu, S.; Yang, H.; Dong, J.; Zhou, J.; Chen, Y. Automatic identification of vulnerable plaque based on flexible neural tree. In Proceedings of the 2018 International Conference on Security, Pattern Analysis, and Cybernetics (SPAC), Jinan, China, 14-17 December 2018; pp. 242-245. [CrossRef]

82. Athanasiou, L.S.; Bourantas, C.V.; Rigas, G.A.; Exarchos, T.P.; Sakellarios, A.I.; Siogkas, P.K.; Papafaklis, M.I.; Naka, K.K.; Michalis, L.K.; Prati, F.; et al. Fully automated calcium detection using optical coherence tomography. In Proceedings of the 2013 35th Annual International Conference of the IEEE Engineering in Medicine and Biology Society (EMBC), Osaka, Japan, 3-7 July 2013; pp. 1430-1433. [CrossRef]

83. Athanasiou, L.S.; Bourantas, C.V.; Rigas, G.; Sakellarios, A.; Exarchos, T.P.; Siogkas, P.K.; Ricciardi, A.; Naka, K.; Papafaklis, M.; Michalis, L.K.; et al. Methodology for fully automated segmentation and plaque characterization in intracoronary optical coherence tomography images. J. Biomed. Opt. 2014, 19, 026009. [CrossRef] [PubMed]

84. Huang, Y.; He, C.; Wang, J.; Miao, Y.; Zhu, T.; Zhou, P.; Li, Z. Intravascular optical coherence tomography image segmentation based on support vector machine algorithm. MCB Mol. Cell. Biomech. 2018, 15, 117-125.

85. Liu, X.; Du, J.; Yang, J.; Xiong, P.; Liu, J.; Lin, F. Coronary Artery Fibrous Plaque Detection Based on Multi-Scale Convolutional Neural Networks. J. Signal Process. Syst. 2020, 92, 325-333. [CrossRef]

86. Dehnavi, S.M.; Babu, M.S.P.; Yazchi, M.; Basij, M. Automatic soft and hard plaque detection in IVUS images: A textural approach. In Proceedings of the 2013 IEEE Conference on Information \& Communication Technologies, Thuckalay, India, 11-12 April 2013; pp. 214-219. [CrossRef]

87. Wang, Z.; Kyono, H.; Bezerra, H.G.; Wang, H.; Gargesha, M.; Alraies, C.; Xu, C.; Schmitt, J.M.; Wilson, D.L.; Costa, M.A.; et al. Semiautomatic segmentation and quantification of calcified plaques in intracoronary optical coherence tomography images. $J$. Biomed. Opt. 2010, 15, 061711. [CrossRef] [PubMed] 
88. Giannoglou, V.G.; Stavrakoudis, D.G.; Theocharis, J.B. IVUS-based characterization of atherosclerotic plaques using feature selection and SVM classification. In Proceedings of the 2012 IEEE 12th International Conference on Bioinformatics \& Bioengineering (BIBE), Larnaca, Cyprus, 11-13 November 2012; pp. 715-720. [CrossRef]

89. Yoshida, Y.; Fujisaku, K.; Sasaki, K.; Yuasa, T.; Shibuya, K. Semi-automatic detection of calcified plaque in coronary CT angiograms with 320-MSCT. In Proceedings of the 2016 24th European Signal Processing Conference (EUSIPCO), Budapest, Hungary, 29 August-2 September 2016; pp. 1703-1707. [CrossRef]

90. Gao, Z.; Guo, W.; Liu, X.; Huang, W.; Zhang, H.; Tan, N.; Hau, W.K.; Zhang, Y.; Liu, H. Automated Detection Framework of the Calcified Plaque with Acoustic Shadowing in IVUS Images. PLoS ONE 2014, 9, e109997. [CrossRef]

91. Taki, A.; Roodaki, A.; Setarehdan, S.K.; Avansari, S.; Unal, G.; Navab, N. An IVUS image-based approach for improvement of coronary plaque characterization. Comput. Biol. Med. 2013, 43, 268-280. [CrossRef]

92. Athanasiou, L.S.; Exarchos, T.P.; Naka, K.K.; Michalis, L.K.; Prati, F.; Fotiadis, D.I. Atherosclerotic plaque characterization in Optical Coherence Tomography images. In Proceedings of the 2011 Annual International Conference of the IEEE Engineering in Medicine and Biology Society, Baltimore, MD, USA, 2-4 June 2011; pp. 4485-4488. [CrossRef]

93. Giannoglou, V.G.; Stavrakoudis, D.G.; Theocharis, J.B.; Petridis, V. Genetic fuzzy rule-based classification systems for tissue characterization of intravascular ultrasound images. In Proceedings of the 2012 IEEE International Conference on Fuzzy Systems, Brisbane, QLD, Australia, 10-15 June 2012; pp. 1-8. [CrossRef]

94. Giannoglou, V.G.; Stavrakoudis, D.; Theocharis, J.B.; Petridis, V. Genetic fuzzy rule based classification systems for coronary plaque characterization based on intravascular ultrasound images. Eng. Appl. Artif. Intell. 2015, 38, 203-220. [CrossRef]

95. Acharya, U.R.; Meiburger, K.M.; Koh, J.E.W.; Vicnesh, J.; Ciaccio, E.J.; Lih, O.S.; Tan, S.K.; Aman, R.R.A.R.; Molinari, F.; Ng, K.H. Automated plaque classification using computed tomography angiography and Gabor transformations. Artif. Intell. Med. 2019, 100, 101724. [CrossRef]

96. Lee, J.; Na Hwang, Y.; Kim, G.Y.; Kwon, J.Y.; Kim, S.M. Automated classification of dense calcium tissues in gray-scale intravascular ultrasound images using a deep belief network. BMC Med. Imaging 2019, 19, 103. [CrossRef] [PubMed]

97. Mahadevi, C.; Sivakumar, S. Performance of Coronary Plaque Feature Extraction and Identification of Plaque Severity for Intravascular Ultrasound B-Mode Images. In International Conference on Mining Intelligence and Knowledge Exploration; Springer: Berlin, Germany, 2019; pp. 221-233.

98. Hwang, Y.N.; Lee, J.H.; Kim, G.Y.; Shin, E.S.; Kim, S.M. Characterization of coronary plaque regions in intravascular ultrasound images using a hybrid ensemble classifier. Comput. Methods Programs Biomed. 2018, 153, 83-92. [CrossRef]

99. Araki, T.; Ikeda, N.; Shukla, D.; Londhe, N.D.; Shrivastava, V.; Banchhor, S.K.; Saba, L.; Nicolaides, A.; Shafique, S.; Laird, J.R.; et al. A new method for IVUS-based coronary artery disease risk stratification: A link between coronary \& carotid ultrasound plaque burdens. Comput. Methods Programs Biomed. 2016, 124, 161-179. [CrossRef] [PubMed]

100. Rezaei, Z.; Selamat, A.; Taki, A.; Rahim, M.S.M.; Kadir, M.R.A.; Penhaker, M.; Krejcar, O.; Kuca, K.; Herrera-Viedma, E.; Fujita, H. Thin Cap Fibroatheroma Detection in Virtual Histology Images Using Geometric and Texture Features. Appl. Sci. 2018, 8, 1632. [CrossRef]

101. Lee, J.; Gharaibeh, Y.; Kolluru, C.; Zimin, V.N.; Dallan, L.A.P.; Kim, J.N.; Bezerra, H.G.; Wilson, D.L. Segmentation of Coronary Calcified Plaque in Intravascular OCT Images Using a Two-Step Deep Learning Approach. IEEE Access 2020, 8 , 225581-225593. [CrossRef]

102. Gessert, N.; Lutz, M.; Heyder, M.; Latus, S.; Leistner, D.M.; Abdelwahed, Y.S.; Schlaefer, A. Automatic Plaque Detection in IVOCT Pullbacks Using Convolutional Neural Networks. IEEE Trans. Med. Imaging 2019, 38, 426-434. [CrossRef] [PubMed]

103. Han, D.; Liu, J.; Sun, Z.; Cui, Y.; He, Y.; Yang, Z. Deep learning analysis in coronary computed tomographic angiography imaging for the assessment of patients with coronary artery stenosis. Comput. Methods Programs Biomed. 2020, 196, 105651. [CrossRef] [PubMed]

104. Jun, T.J.; Kang, S.-J.; Lee, J.-G.; Kweon, J.; Na, W.; Kang, D.; Kim, D.; Kim, D.; Kim, Y.-H. Automated detection of vulnerable plaque in intravascular ultrasound images. Med. Biol. Eng. Comput. 2019, 57, 863-876. [CrossRef]

105. Ughi, G.J.; Adriaenssens, T.; Sinnaeve, P.; Desmet, W.; D’hooge, J. Automated tissue characterization of in vivo atherosclerotic plaques by intravascular optical coherence tomography images. Biomed. Opt. Express 2013, 4, 1014-1030. [CrossRef] [PubMed]

106. Kolluru, C.; Prabhu, D.; Gharaibeh, Y.; Bezerra, H.; Guagliumi, G.; Wilson, D. Deep neural networks for A-line-based plaque classification in coronary intravascular optical coherence tomography images. J. Med. Imaging 2018, 5, 044504. [CrossRef] [PubMed]

107. Athanasiou, L.S.; Olender, M.L.; Hernandez, J.M.D.L.T.; Ben-Assa, E.; Edelman, E.R. A deep learning approach to classify atherosclerosis using intracoronary optical coherence tomography. In Medical Imaging 2019: Computer-Aided Diagnosis; International Society for Optics and Photonics: Bellingham, WA, USA, 2019; Volume 109500N. [CrossRef]

108. Zreik, M.; van Hamersvelt, R.W.; Wolterink, J.M.; Leiner, T.; Viergever, M.A.; Isgum, I. Automatic Detection and Characterization of Coronary Artery Plaque and Stenosis Using a Recurrent Convolutional Neural Network in Coronary CT Angiography; European Society of Radiology: Vienna, Austria, 2018.

109. Zreik, M.; Van Hamersvelt, R.W.; Wolterink, J.M.; Leiner, T.; Viergever, M.A.; Isgum, I. A Recurrent CNN for Automatic Detection and Classification of Coronary Artery Plaque and Stenosis in Coronary CT Angiography. IEEE Trans. Med. Imaging 2019, 38, 1588-1598. [CrossRef] 
110. Liu, J.; Jin, C.; Feng, J.; Du, Y.; Lu, J.; Zhou, J. A Vessel-Focused 3D Convolutional Network for Automatic Segmentation and Classification of Coronary Artery Plaques in Cardiac CTA. In International Workshop on Statistical Atlases and Computational Models of the Heart; Springer: Berlin, Germany, 2018; pp. 131-141.

111. Acharya, U.R.; Meiburger, K.M.; Koh, J.E.W.; Ciaccio, E.J.; Vicnesh, J.; Tan, S.K.; Wong, J.H.D.; Aman, R.R.A.R.; Ng, K.H. Automated detection of calcified plaque using higher-order spectra cumulant technique in computer tomography angiography images. Int. J. Imaging Syst. Technol. 2020, 30, 285-297. [CrossRef]

112. Filho, E.D.S.; Saijo, Y.; Tanaka, A.; Yambe, T.; Li, S.; Yoshizawa, M. Automated Calcification Detection and Quantification in Intravascular Ultrasound Images by Adaptive Thresholding. In Proceedings of the World Congress on Medical Physics and Biomedical Engineering, Seoul, Korea, 27 August-1 September 2006; pp. 1421-1425.

113. Sofian, H.; Ng, A.; Than, J.; Mohamad, S.; Noor, N.M. Calcification boundary detection in coronary artery using intravascular ultrasound images. In Proceedings of the TENCON 2017-2017 IEEE Region 10 Conference, Penang, Malaysia, 5-8 November 2017; pp. 2835-2839. [CrossRef]

114. Roy, A.G.; Conjeti, S.; Carlier, S.G.; Houissa, K.; König, A.; Dutta, P.K.; Laine, A.F.; Navab, N.; Katouzian, A.; Sheet, D. Multiscale distribution preserving autoencoders for plaque detection in intravascular optical coherence tomography. In Proceedings of the 2016 IEEE 13th International Symposium on Biomedical Imaging (ISBI), Prague, Czech Republic, 13-16 April 2016; pp. 1359-1362. [CrossRef]

115. Sofian, H.; Ming, J.T.C.; Mohamad, S.; Noor, N.M. Calcification Detection Using Deep Structured Learning in Intravascular Ultrasound Image for Coronary Artery Disease. In Proceedings of the 2018 2nd International Conference on BioSignal Analysis, Processing and Systems (ICBAPS), Kuching, Malaysia, 24-26 July 2018; pp. 47-52. [CrossRef]

116. Sofian, H.; Ming, J.T.C.; Muhammad, S.; Noor, N.M. Calcification detection using convolutional neural network architectures in intravascular ultrasound images. J. Electr. Eng. Comput. Sci. 2019, 17, 1313-1321. [CrossRef]

117. De Vos, B.D.; Wolterink, J.M.; Leiner, T.; De Jong, P.A.; Lessmann, N.; Isgum, I. Direct Automatic Coronary Calcium Scoring in Cardiac and Chest CT. IEEE Trans. Med. Imaging 2019, 38, 2127-2138. [CrossRef] [PubMed]

118. Zeleznik, R.; Foldyna, B.; Eslami, P.; Weiss, J.; Alexander, I.; Taron, J.; Parmar, C.; Alvi, R.M.; Banerji, D.; Uno, M.; et al. Deep convolutional neural networks to predict cardiovascular risk from computed tomography. Nat. Commun. 2021, 12, 715. [CrossRef] [PubMed]

119. Balocco, S.; González, M.; Nanculef, R.; Radeva, P.; Thomas, G. Calcified Plaque Detection in IVUS Sequences: Preliminary Results Using Convolutional Nets. In International Workshop on Artificial Intelligence and Pattern Recognition; Springer: Berlin/Heidelberg, Germany, 2018; pp. 34-42.

120. Liu, S.; Neleman, T.; Hartman, E.M.; Ligthart, J.M.; Witberg, K.T.; van der Steen, A.F.; Wentzel, J.J.; Daemen, J.; van Soest, G. Automated Quantitative Assessment of Coronary Calcification Using Intravascular Ultrasound. Ultrasound Med. Biol. 2020, 46, 2801-2809. [CrossRef]

121. Li, Y.-C.; Shen, T.-Y.; Chen, C.-C.; Chang, W.-T.; Lee, P.-Y.; Huang, C.-C.J. Automatic Detection of Atherosclerotic Plaque and Calcification from Intravascular Ultrasound Images by Using Deep Convolutional Neural Networks. IEEE Trans. Ultrason. Ferroelectr. Freq. Control 2021, 68, 1762-1772. [CrossRef]

122. Sun, Q.; Yang, G.; Shu, H. Calcified coronary plaques detection in CTA based-on automatic scale selection and fuzzy C means. In Proceedings of the 2016 International Conference on Machine Learning and Cybernetics (ICMLC), Jeju, Korea, 10-13 July 2016; pp. 807-813. [CrossRef]

123. Mirunalini, P.; Aravindan, C.; Nambi, A.T.; Poorvaja, S.; Priya, V.P. Segmentation of Coronary Arteries from CTA axial slices using Deep Learning techniques. In Proceedings of the TENCON 2019-2019 IEEE Region 10 Conference (TENCON), Kochi, India, 17-20 October 2019; pp. 2074-2080. [CrossRef]

124. Mirunalini, P.; Aravindan, C.; Jaisakthi, S.M. Automatic stenosis detection using SVM from CTA projection images. Multimed. Syst. 2019, 25, 83-93. [CrossRef]

125. Chow, B.J.; Abraham, A.; Wells, G.A.; Chen, L.; Ruddy, T.D.; Yam, Y.; Govas, N.; Galbraith, P.D.; Dennie, C.; Beanlands, R.S. Diagnostic accuracy and impact of computed tomographic coronary angiography on utilization of invasive coronary angiography. Circ. Cardiovasc. Imaging 2009, 2, 16-23. [CrossRef]

126. Eckert, J.; Schmidt, M.; Magedanz, A.; Voigtländer, T.; Schmermund, A. Coronary CT angiography in managing atherosclerosis. Int. J. Mol. Sci. 2015, 16, 3740-3756. [CrossRef]

127. Leber, A.W.; Knez, A.; von Ziegler, F.; Becker, A.; Nikolaou, K.; Paul, S.; Wintersperger, B.; Reiser, M.; Becker, C.R.; Steinbeck, G.; et al. Quantification of obstructive and nonobstructive coronary lesions by 64-slice computed tomography: A comparative study with quantitative coronary angiography and intravascular ultrasound. J. Am. Coll. Cardiol. 2005, 46, 147-154. [CrossRef] [PubMed]

128. Alkadhi, H.; Scheffel, H.; Desbiolles, L.; Gaemperli, O.; Stolzmann, P.; Plass, A.; Goerres, G.W.; Luescher, T.F.; Genoni, M.; Marincek, B.; et al. Dual-source computed tomography coronary angiography: Influence of obesity, calcium load, and heart rate on diagnostic accuracy. Eur. Heart J. 2008, 29, 766-776. [CrossRef]

129. Hoffmann, U.; Bamberg, F.; Chae, C.U.; Nichols, J.H.; Rogers, I.S.; Seneviratne, S.K.; Truong, Q.A.; Cury, R.C.; Abbara, S.; Shapiro, M.D.; et al. Coronary computed tomography angiography for early triage of patients with acute chest pain: The ROMICAT (Rule Out Myocardial Infarction using Computer Assisted Tomography) trial. J. Am. Coll. Cardiol. 2009, 53, 1642-1650. [CrossRef] 
130. Song, F.X.; Zhou, J.; Zhou, J.J.; Shi, Y.X.; Zeng, M.S.; Zhang, Z.Y.; Lv, P.; Sheng, R.F. The diagnosis of coronary plaque stability by multi-slice computed tomography coronary angiography. J. Thorac. Dis. 2018, 10, 2365-2376. [CrossRef]

131. Sehovic, S. Diagnostic Capabilities of 64 Slice CT Coronography Compared to Classic in Coronary Disease Detection. Acta Inform. Med. 2013, 21, 208-210. [CrossRef]

132. Mowatt, G.; Cook, J.; Hillis, G.S.; Walker, S.; Fraser, C.; Jia, X.; Waugh, N. 64-Slice computed tomography angiography in the diagnosis and assessment of coronary artery disease: Systematic review and meta-analysis. Heart 2008, 94, 1386-1393. [CrossRef]

133. Wong, D.T.; Ko, B.S.; Cameron, J.D.; Nerlekar, N.; Leung, M.C.; Malaiapan, Y.; Crossett, M.; Leong, D.P.; Worthley, S.G.; Troupis, J.; et al. Transluminal attenuation gradient in coronary computed tomography angiography is a novel noninvasive approach to the identification of functionally significant coronary artery stenosis: A comparison with fractional flow reserve. J. Am. Coll. Cardiol. 2013, 61, 1271-1279. [CrossRef]

134. Yoon, Y.E.; Choi, J.H.; Kim, J.H.; Park, K.W.; Doh, J.H.; Kim, Y.J.; Koo, B.K.; Min, J.K.; Erglis, A.; Gwon, H.C.; et al. Noninvasive diagnosis of ischemia-causing coronary stenosis using CT angiography: Diagnostic value of transluminal attenuation gradient and fractional flow reserve computed from coronary CT angiography compared to invasively measured fractional flow reserve. JACC Cardiovasc. Imaging 2012, 5, 1088-1096.

135. Gonzalez, J.A.; Lipinski, M.J.; Flors, L.; Shaw, P.W.; Kramer, C.M.; Salerno, M. Meta-analysis of diagnostic performance of coronary computed tomography angiography, computed tomography perfusion, and computed tomography-fractional flow reserve in functional myocardial ischemia assessment versus invasive fractional flow reserve. Am. J. Cardiol. 2015, 116, 1469-1478. [CrossRef]

136. Baumann, S.; Renker, M.; Hetjens, S.; Fuller, S.R.; Becher, T.; Loßnitzer, D.; Lehmann, R.; Akin, I.; Borggrefe, M.; Lang, S.; et al. Comparison of coronary computed tomography angiography-derived vs invasive fractional flow reserve assessment. Meta-analysis with subgroup evaluation of intermediate stenosis. Acad. Radiol. 2016, 23, 1402-1411. [CrossRef]

137. Fischer, C.; Hulten, E.; Belur, P.; Smith, R.; Voros, S.; Villines, T.C. Coronary CT angiography versus intravascular ultrasound for estimation of coronary stenosis and atherosclerotic plaque burden: A meta-analysis. J. Cardiovasc. Comput. Tomogr. 2013, 7 , 256-266. [CrossRef]

138. Kolossváry, M.; Kellermayer, M.; Merkely, B.; Maurovich-Horvat, P. Cardiac Computed Tomography Radiomics: A Comprehensive Review on Radiomic Techniques. J. Thorac. Imaging 2018, 33, 26-34. [CrossRef] [PubMed]

139. Kigka, V.I.; Sakellarios, A.; Kyriakidis, S.; Rigas, G.; Athanasiou, L.; Siogkas, P.; Tsompou, P.; Loggitsi, D.; Benz, D.C.; Buechel, R.; et al. A three-dimensional quantification of calcified and non-calcified plaques in coronary arteries based on computed tomography coronary angiography images: Comparison with expert's annotations and virtual histology intravascular ultrasound. Comput. Biol. Med. 2019, 113, 103409. [CrossRef] [PubMed]

140. Wu, W.; Zhang, J.; Xie, H.; Zhao, Y.; Zhang, S.; Gu, L. Automatic detection of coronary artery stenosis by convolutional neural network with temporal constraint. Comput. Biol. Med. 2020, 118, 103657. [CrossRef] 